\title{
Effect of Foot Reflexology Intervention on Depression, Anxiety, and Sleep Quality in Adults: A Meta-Analysis and Metaregression of Randomized Controlled Trials
}

\author{
Wei-Li Wang, ${ }^{1}$ Hao-Yuan Hung, ${ }^{2,3,4}$ Ying-Ren Chen, ${ }^{5,6}$ Kuang-Huei Chen, ${ }^{1}$ \\ Szu-Nian Yang, ${ }^{1,7,8}$ Chi-Ming Chu, ${ }^{9}$ and Yuan-Yu Chan $\mathbb{1}^{1,10}$ \\ ${ }^{1}$ Department of Psychiatry, Taoyuan Armed Forces General Hospital, Taoyuan, Taiwan \\ ${ }^{2}$ Department of Pharmacology, National Defense Medical Center, Taipei, Taiwan \\ ${ }^{3}$ Department of Pharmacy Practice, Tri-Service General Hospital, National Defense Medical Center, Taipei, Taiwan \\ ${ }^{4}$ Graduate Institute of Medical Sciences, National Defense Medical Center, Taipei, Taiwan \\ ${ }^{5}$ Department of Nursing, Taoyuan Armed Forces General Hospital, Taoyuan, Taiwan \\ ${ }^{6}$ Graduate Institute of Nursing, College of Nursing, Taipei Medical University, Taipei, Taiwan \\ ${ }^{7}$ Tri-Service General Hospital, Beitou Branch, National Defense Medical Center, Taipei, Taiwan \\ ${ }^{8}$ Graduate Institute of Health and Welfare Policy, School of Medicine, National Yang-Ming University, Taipei, Taiwan \\ ${ }^{9}$ Department of Epidemiology, School of Public Health, National Defense Medical Center, Taipei, Taiwan \\ ${ }^{10}$ Department of Psychology, Chung Yuan Christian University, Taoyuan, Taiwan
}

Correspondence should be addressed to Yuan-Yu Chan; xaviorchan@gmail.com

Received 31 January 2020; Revised 20 August 2020; Accepted 5 September 2020; Published 15 September 2020

Academic Editor: Gerhard Litscher

Copyright (C) 2020 Wei-Li Wang et al. This is an open access article distributed under the Creative Commons Attribution License, which permits unrestricted use, distribution, and reproduction in any medium, provided the original work is properly cited.

\begin{abstract}
Objectives. The aim of this study was to conduct a systematic review, meta-analysis, and metaregression to determine the current best available evidence of the efficacy and safety of foot reflexology for adult depression, anxiety, and sleep quality. Methods. Electronic databases (PubMed, ClinicalKey, ScienceDirect, EMBASE, PsycINFO, and the Cochrane Library) were searched till August, 10, 2020, and the validity of the eligible studies was critically appraised. Randomized controlled trials comparing foot reflexology groups with control groups for adult depression, anxiety, and sleep quality were included. Twenty-six eligible studies were included to assess the effect of foot reflexology intervention on the reducing symptoms of depression and anxiety and improving quality of sleep, respectively, as the primary outcome. Results. Twenty-six randomized controlled trials involving 2,366 participants met the inclusion criteria. The meta-analyses showed that foot reflexology intervention significantly improved adult depression (Hedges' $g=-0.921 ; 95 \% \mathrm{CI}:-1.246$ to $-0.595 ; P<0.001$ ), anxiety (Hedges' $g=-1.237 ; 95 \%$ CI -1.682 to -0.791 ; $P<0.001$ ), and sleep quality (Hedges' $g=-1.665 ; 95 \% \mathrm{CI}-2.361$ to $-0.970 ; P<0.001$ ). Metaregression reveals that an increase in total foot reflexology time $(P=0.002)$ and duration $(P=0.01)$ can significantly improve sleep quality. Conclusions. Foot reflexology may provide additional nonpharmacotherapy intervention for adults suffering from depression, anxiety, or sleep disturbance. However, high quality and rigorous design RCTs in specific population, along with an increase in participants, and a long-term follow-up are recommended in the future.
\end{abstract}

\section{Introduction}

Foot reflexology is a systemic practice in which a practitioner applies some pressure to any pressure points on the feet to stimulate the body and provide health benefits to different parts of the body. Foot reflexology is commonly practiced as a complementary therapy and is one of the nonpharmacological therapies to alleviate our mental, emotional, and spiritual health, while improving the quality of our life [1].

Foot reflexology is a reflexology intervention that has been applied in different cultures around the world for 
thousands of years. It is defined as a type of therapy that is based on the stimulation of the nerves and circulatory system of the body in which all the reflexology points, corresponding to different parts of the human body, are considered [2]. It is still ambiguous regarding the mechanism behind the function of foot reflexology, but it certainly has been shown to have potent physiological and psychological effects, perhaps attributed to the relaxation derived from the placebo effect, the therapeutic communication techniques, and impact of touching behavior. The explication for the mechanism of action in foot reflexology is based on the theory that helps to equilibrate the energy in the whole physical structure $[3,4]$. Currently, the most promising theory suggests that the benefits of foot reflexology may be caused by modulating our autonomic nervous system [5]. The effects are well known to relieve the psychological symptoms of stress by reducing anxiety and muscle tension [6], calming our mood [7], improving the quality of sleep [8], and facilitating the feeling of well-being [9]. Pharmacological treatment of prevalent symptoms such as depression, anxiety, and sleep disturbance may contribute to the high strain on the body, creating additional side effects [10]. Foot reflexology provides an advantage to certain groups and generally does not cause any damaging effects during certain medical circumstances. Every person's body circumstance is unique, so outcomes from foot reflexology intervention could differ from one person to another [11].

The previous systematic review had reported physiological and biochemical outcomes associated with foot reflexology intervention [12]. However, there are insufficient number of evidence-based studies that expound the effects of foot reflexology on improving our psychological symptoms such as depression, anxiety, and sleep disturbance. To our knowledge, this is the first systematic review and metaanalysis on the psychological effect of foot reflexology and to identify the possible related factors of foot reflexology in adult participants.

\section{Methods}

2.1. Reporting Standards. The present study was designed, executed, and adopted in accordance with the Preferred Reporting Items for Systematic Reviews and Meta-analyses (PRISMA) statement guidelines [13] and the suggestions by the Cochrane Collaboration [14]. The protocol for this systematic review and meta-analysis is registered with PROSPERO under registration number CRD42020162545.

\subsection{Eligibility Criteria}

2.2.1. Types of Studies. Randomized controlled trials (RCTs), randomized crossover trials, and cluster randomized trials all met our inclusion criteria. The language of the studies was restricted to English.

2.2.2. Types of Participants. Adults aged 18 years or older without restrictions on sociodemography, race, gender, or health status were participants. All studies that reported on depression, anxiety, or sleep quality were included. There was no restriction on the baseline for these.

2.2.3. Types of Interventions. No further restrictions were made regarding the foot reflexology zone, constitution, length, frequency, or duration of intervention programs. Studies on cointerventions that included foot reflexology as a part of multimodal interventions were excluded because it would be hard to evaluate the influence of foot reflexology from additional modalities. Shame intervention, care-asusual, nontreatment waitlists, and psychoeducation about depression, anxiety, or sleep hygiene information are considered as the nonactive control group.

2.2.4. Types of Outcome Measures. Studies include, at least, one efficacy outcome index related to depression, anxiety, and sleep quality. Our primary outcome measures of this study were depression, anxiety, and sleep disturbance. Data are presented both at baseline and after intervention. We take various clinical outcomes that were informed in the selected RCTs to show improvements in the symptoms of depression, anxiety, and sleep disturbance into consideration. No restrictions were set on the scales of measurement used to evaluate these outcomes because a wide variety of measures in the outcomes were applied in the studies.

Our secondary outcome of this study was intervention safety, which assessed the number of participants with adverse events, including serious adverse events or nonserious events. Adverse events resulting in death, life-threatening situations, hospitalization, disability or permanent damage, congenital anomaly/birth defect, or the need for medical or surgical intervention to prevent the aforementioned outcomes were defined as serious [15]. All other adverse events were regarded as nonserious.

2.3. Search Methods. The following electronic databases were searched from their inception to August 10, 2020: PubMed, ClinicalKey, ScienceDirect, EMBASE, PsycINFO, and the Cochrane Library. The search was performed using the keywords "foot reflexology," "depression," "anxiety," and "sleep quality." The complete search through PubMed was conducted using the medical subjective headings (MeSHs) as follows: (foot reflexology [MeSH] OR foot reflexology [Title/ Abstract] OR foot massage [MeSH] OR foot massage [Title/ Abstract] OR reflexology [MeSH] OR reflexology [Title/ Abstract]) AND (depression [MeSH] OR depression [Title/ Abstract] OR depressive disorder [MeSH] OR depressive disorder [Title/Abstract] OR anxiety [MeSH] OR anxiety [Title/Abstract] OR anxiety disorder [MeSH] OR anxiety disorder [Title/Abstract] sleep quality [MeSH] OR sleep quality [Title/Abstract] OR insomnia [MeSH] OR insomnia [Title/Abstract] OR sleep disturbance [MeSH] OR sleep disturbance [Title/Abstract]). The search strategy was adapted for each database as necessary.

The references of our retrieved studies and previous systematic reviews were manually screened to ensure a comprehensive search. Additionally, Google Scholar search 
engine was utilized to identify extra articles that had not yet been included in the previously mentioned electronic databases.

The titles and abstracts were scanned independently by two independent reviewers. When there was disagreement on eligibility, we discussed with a third reviewer to reach a consensus.

2.4. Data Extraction Method. Two reviewers independently extracted general information from the aforementioned selected publications on design and study sample (e.g., article setting, first author's name/year of publication, and origin), participants (e.g., mean age, gender, clinical characteristics, comorbid conditions, and the number of participants), interventions (e.g., foot reflexology zone, components, frequency, duration, and length of foot reflexology), control interventions (e.g., shame intervention, treatment-as-usual, and waitlist), and outcomes (e.g., outcome measurement tools, measured outcomes, adherence, eventual follow-up time, and adverse events). Any disagreements between the two reviewers should refer to the third reviewer's opinion.

2.5. Quality and Risk-of-Bias Assessments. Two reviewers independently assessed the risk of bias in each study. There were seven domains of assessment for the risk of bias including the following: (1) random sequence generation, (2) allocation concealment, (3) blinding of participants and personnel, (4) blinding of outcome assessment, (5) incomplete outcome data, (6) selective reporting, and (7) other biases using the Cochrane Systematic Review Manual riskof-bias assessment tool [16]. These rates were then labeled as "low risk," "high risk," or "unclear risk" of bias. A risk-ofbias table was completed for each included study. To improve accuracy, any disagreements would refer to a third reviewer's opinion.

2.6. Data Synthesis and Statistical Analysis. Meta-analysis was performed using Comprehensive Meta-Analysis Software. The random-effects model was used to calculate the pooled effect size of the included studies. Hedges' $g$ was calculated to determine the effect size [17]. The effect size represents the difference between two groups in the number of standard deviations. An effect size of 0.2-0.49 was considered a small effect, $0.5-0.79$ was a moderate effect, 0.8 and higher was a large effect [18]. The meta-analysis results were expressed as the pool effect, with corresponding 95\% and $P$ value. The heterogeneity data were evaluated using a random-effects model because it accommodated the possibility that the underlying effect differed across studies.

2.7. Assessment of Heterogeneity. Heterogeneity between studies was evaluated using the $\mathrm{I}^{2}$ statistic with a cutoff point $>50 \%$, and a $P$ value $\leq 0.1$ was regarded as a significant degree of heterogeneity. The most common $I^{2}$ scale considered values lower than $25 \%$ as low heterogeneity; values between $25 \%-50 \%$ as mean heterogeneity; values between $50 \%-74 \%$ as substantial heterogeneity; and values between $75 \%-100 \%$ as considerable heterogeneity [14]. Random effects of univariate and multivariate meta-regressions were used to explore the source of heterogeneity if $I^{2}>50 \%$ and $P$ value $\leq 0.1$.

2.8. Moderator Analyses. We performed subgroup metaanalysis and metaregression analysis to examine possible sources of heterogeneity and survey the possible confounding effects of clinical variables. The subgroup analysis produced prespecified covariates, including outcome measurement instruments, study quality, and participant details. Additionally, continuous covariates were obtained from the metaregression analysis to investigate whether relationships were linear and consistent with the results of the categorical analysis. A metaregression model was performed to test between-subgroup interaction, and a $P$ value $<0.05$ indicated a significant difference.

2.9. Risk of Publication Bias. Publication bias was explored if there were up to ten eligible trials included in the metaanalysis. Funnel plots generated using Comprehensive Meta-Analysis Software were estimated from individual studies against each study's standard error. The presence of asymmetry with a visual inspection in funnel plots was considered potentially indicative of publication bias [19]. Potential publication bias was tested using the calculation of Egger's regression method, with $P$ values $<0.05$ suggesting the presence of bias.

\section{Results}

3.1. Research Material. The search strategy identified 959 research articles through electronic databases. A total of 912 records were excluded after removal of duplicates and screening of abstracts and titles. Then, all full-text articles were evaluated for eligibility, and 21 records were excluded for reasons such as they were not randomized [20-30], did not include relevant outcomes [31-33], included foot reflexology as a part of a multimodal intervention [34-38], lacked adequate control group [39], has yet to be officially published [40]. Finally, 26 remaining articles with 2,366 participants were investigated by qualitative analyses. All articles were published in English. The flowchart of the study selection process is presented in Figure 1.

3.2. Characteristics of Eligible Studies. The characteristics of the 26 eligible studies are presented in Tables 1 and 2. All study assessed outcomes are listed directly at the end of foot reflexology intervention. Among the 26 RCTs selected for our study efficacy, the psychological symptoms of depression, anxiety, and sleep quality, respectively, were assessed as the primary outcome. Our studies were conducted in Iran, Turkey, Taiwan, South Korea, Japan, and Israel. All of the included studies were published between 2011 and 2020. The sample sizes ranged from 50 to 189 individuals (total 2,366 participants). The average age ranged from 27 to 72 years, 


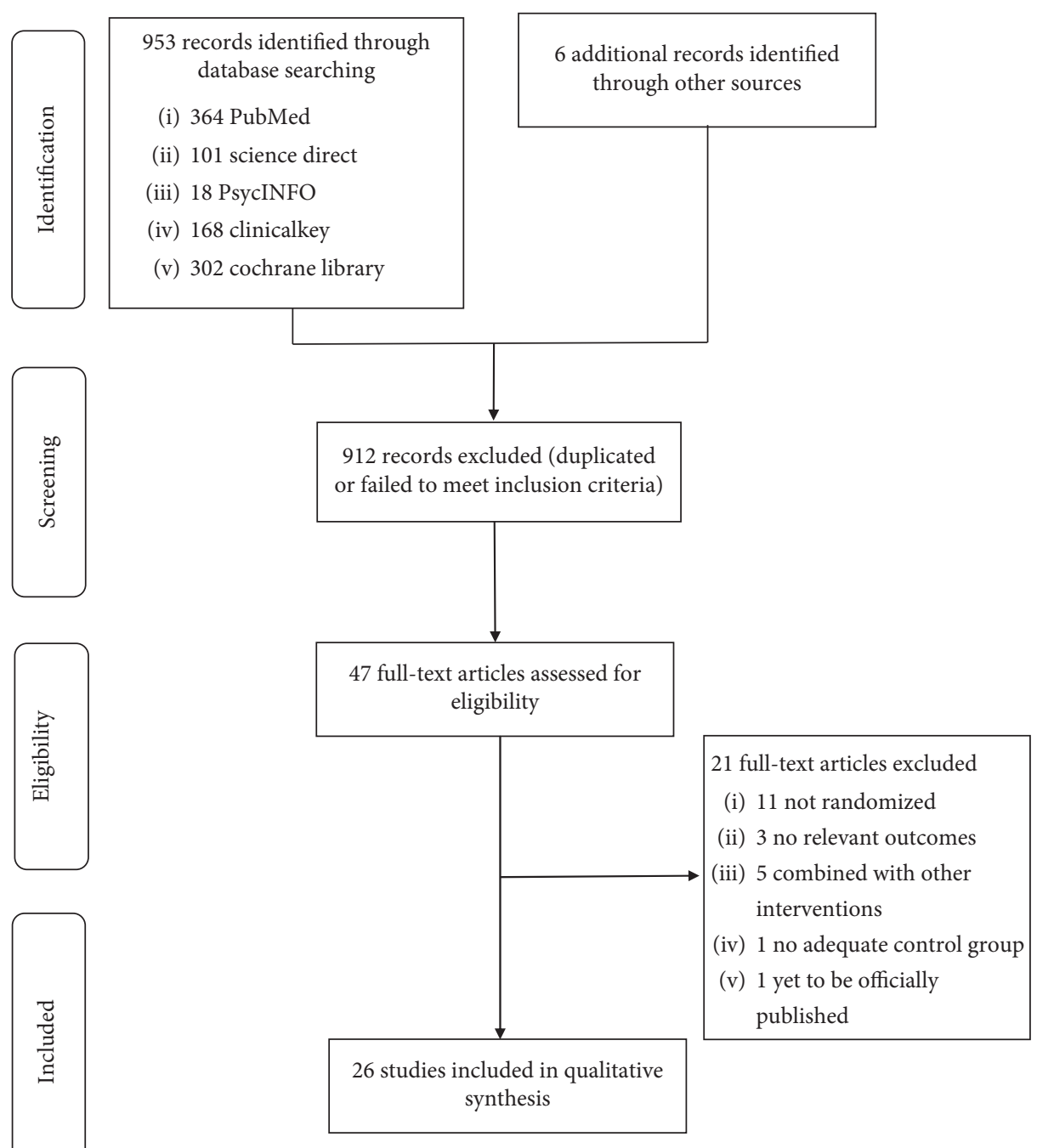

FIGURE 1: Flowchart of the results of the literature search.

and all the participants were adults (age $>18$ years). In each study, foot reflexology intervention for one session lasted between 10-60 min (total treatment sessions ranged from 1 to 18 in each study). Also, the total treatment periods ranged from 1 to 8 weeks. Adherence to the foot reflexology was reported in all studies as the percentage in foot reflexology session dropouts. Adherence was $>90 \%$ in all studies. Evidence of safety issue evaluation was limited because only a few studies report safety-related adverse effects as the secondary outcome. Most of the included studies failed to report on this aspect making research difficult.

\subsection{Risk of Bias}

3.3.1. Quality of Methods. Risk-of-bias assessment is shown in Table 3. Twenty-six studies were assessed as high or unclear risk of bias in at least one of the domains. All studies reviewed stated they were randomized, whether or not this is true remains uncertain as eight studies did not show their content and method of random sequencing [7, 41-47]. A small proportion of studies were low risk due to the state of detailed randomization and allocation methods [20, 48-52]. Most studies yielded no data material on bias concealment. One study had insufficient data on attrition rates [44]. We found no included studies with potential bias in the domain of selective reporting. Other potential sources of bias were high in 9 RCTs due to poor compliance, incomplete outcome data, small sample size, or obvious baseline differences [41-46, 48, 52, 53].

3.3.2. Publication Bias. The funnel plots on the efficacy of foot reflexology for psychological symptoms for depression, anxiety, and sleep disturbance were executed including 4 RCTs, 16 RCTs, 10 RCTs, respectively. The visual inspection of the funnel plots indicated some risk of publication bias for the effects of foot reflexology only in the domain of anxiety symptoms (shown in Figure 2). Those plots examined were shown to be asymmetrical, suggesting the possible risk of publication bias. Moreover, results of Egger's regression test indicated no significant publication bias (Egger test intercept $=-7.32 ; P=0.11)$. Therefore, the overall population effect size was likely to be relatively robust. 
TABLE 1: Characteristics of included studies.

\begin{tabular}{|c|c|c|c|c|c|c|}
\hline $\begin{array}{l}\text { Authors, year, } \\
\text { country }\end{array}$ & $\begin{array}{c}\text { Main characteristics } \\
\text { of studied } \\
\text { population }\end{array}$ & $\begin{array}{c}\text { Sample } \\
\text { characteristics } \\
\text { (sample size, } \\
\text { mean age) }\end{array}$ & Sex difference & $\begin{array}{l}\text { Intervention } \\
\text { group. vs. } \\
\text { comparison } \\
\text { group }\end{array}$ & $\begin{array}{c}\text { Outcome } \\
\text { measurement tools }\end{array}$ & Outcomes \\
\hline $\begin{array}{l}\text { Valizadeh et al., } \\
\text { 2015, Iran }\end{array}$ & $\begin{array}{l}\text { Participants between } \\
\text { the age of } 60-75 \mathrm{y} / \mathrm{o} \\
\text { independently } \\
\text { performing daily } \\
\text { activities and having } \\
\text { mental health based } \\
\text { on health records } \\
\text { available in the } \\
\text { health center }\end{array}$ & $\begin{array}{c}69, \mathrm{G} 1=23, \\
\mathrm{G} 2=23, \mathrm{G} 3=23 \\
\text { mean age: } \\
\mathrm{G} 1=66.82 \mathrm{y} / \mathrm{o} \\
(\mathrm{SD}=4.80), \\
\mathrm{G} 2=67.69 \mathrm{y} / \mathrm{o} \\
(\mathrm{SD}=4.28), \\
\mathrm{G} 3=66.82 \mathrm{y} / \mathrm{o} \\
(\mathrm{SD}=3.84)\end{array}$ & $\begin{array}{l}\text { Male: } 69 \\
\text { Female: } 0\end{array}$ & $\begin{array}{c}\mathrm{G} 1=\text { foot } \\
\text { reflexology } \\
\mathrm{G} 2=\text { footbath } \\
\mathrm{G} 3=\text { control } \\
\text { group }\end{array}$ & $\begin{array}{c}\text { Pittsburgh Sleep } \\
\text { Quality Index (PSQI) }\end{array}$ & $\begin{array}{c}\text { The total score of } \\
\text { PSQI improved: } \\
\text { no statistically } \\
\text { significant } \\
\text { finding G1 vs. G3 } \\
(P<0.05) \\
\mathrm{G} 1=6.08(5.27), \\
\mathrm{G} 1^{*}=3.91(4.04) \\
\mathrm{G} 3=4.69(0.51), \\
\mathrm{G}^{*}=5.69(3.08)\end{array}$ \\
\hline $\begin{array}{l}\text { Lee et al., 2011, } \\
\text { Taiwan }\end{array}$ & $\begin{array}{l}\text { Postpartum women } \\
\text { have given birth } \\
\text { vaginally without } \\
\text { postpartum } \\
\text { complications and } \\
\text { concurrent medical } \\
\text { conditions with poor } \\
\text { sleep condition } \\
\text { (PSQI } \geq 5)\end{array}$ & $\begin{array}{c}68, \mathrm{G} 1=34, \\
\mathrm{G} 2=34 \text { mean age: } \\
\mathrm{G} 1=32.0 \mathrm{y} / \mathrm{o} \\
(\mathrm{SD}=2.8) \\
\mathrm{G} 2=31.2 \mathrm{y} / \mathrm{o} \\
(\mathrm{SD}=2.8)(3 \mathrm{drop} \\
\text { out })\end{array}$ & $\begin{array}{c}\text { Male: } 0 \\
\text { Female: } 68\end{array}$ & $\begin{array}{c}\mathrm{G} 1=\text { foot } \\
\text { reflexology } \\
\mathrm{G} 2=\text { control } \\
\text { group }\end{array}$ & $\begin{array}{c}\text { Pittsburgh Sleep } \\
\text { Quality Index (PSQI) }\end{array}$ & $\begin{array}{c}\text { The total score of } \\
\text { PSQI improved: } \\
\text { G1 vs. G2 } \\
(P<0.001) \\
\mathrm{G} 1=9.94(2.61), \\
\mathrm{G}^{*}=3.97(1.26) \\
\mathrm{G} 2=9.45(2.59), \\
\mathrm{G} 2^{*}=6.24(1.68)\end{array}$ \\
\hline $\begin{array}{l}\text { Bakir et al., } \\
\text { 2018, Turkey }\end{array}$ & $\begin{array}{c}\text { Voluntary } \\
\text { participants aged } \\
\geq 18 \text { y/o diagnosed } \\
\text { with rheumatoid } \\
\text { arthritis, at least, } 1 \\
\text { year with VAS-Pain } \\
\text { (visual analogue } \\
\text { scale for pain) of } 4 \text { or } \\
\text { greater }\end{array}$ & $\begin{array}{c}60, \mathrm{G} 1=30, \\
\mathrm{G} 2=30 \text { mean age: } \\
\mathrm{G} 1=50.83 \mathrm{y} / \mathrm{o} \\
(\mathrm{SD}=12.0) \\
\mathrm{G} 2=49.50 \mathrm{y} / \mathrm{o} \\
(\mathrm{SD}=16.4)(5 \\
\text { drop out })\end{array}$ & $\begin{array}{l}\text { Male: } 14 \\
\text { Female: } 46\end{array}$ & $\begin{array}{c}\mathrm{G} 1=\text { foot } \\
\text { reflexology } \\
\mathrm{G} 2=\text { control } \\
\text { group }\end{array}$ & $\begin{array}{c}\text { Pittsburgh Sleep } \\
\text { Quality Index (PSQI) }\end{array}$ & $\begin{array}{c}\text { The total score of } \\
\text { PSQI improved: } \\
\text { G1 vs. G2 } \\
(P=0.001) \\
\text { G1 }=16.20(3.70), \\
\text { G1 }{ }^{*}=13.16 \\
(3.57) \mathrm{G} 2=16.75 \\
(3.64), \\
\mathrm{G}^{*}=19.03 \\
(3.05)\end{array}$ \\
\hline $\begin{array}{l}\text { Unal et al., } \\
\text { 2016, Turkey }\end{array}$ & $\begin{array}{c}\text { Patients between the } \\
\text { age of } 18-60 \text { y/o who } \\
\text { received } \\
\text { hemodialysis therapy } \\
\text { twice a week without } \\
\text { any communication } \\
\text { problems }\end{array}$ & $\begin{array}{c}105, \mathrm{G} 1=35, \\
\mathrm{G} 2=35, \mathrm{G} 3=35 \\
\text { mean age: } \\
\mathrm{G} 1=51.74 \mathrm{y} / \mathrm{o} \\
(\mathrm{SD}=12.2), \\
\mathrm{G} 2=53.89 \mathrm{y} / \mathrm{o} \\
(\mathrm{SD}=13.1), \\
\mathrm{G} 3=54.33 \mathrm{y} / \mathrm{o} \\
(\mathrm{SD}=12.9)\end{array}$ & $\begin{array}{l}\text { Male: } 55 \\
\text { Female: } 50\end{array}$ & $\begin{array}{l}\mathrm{G} 1=\text { foot } \\
\text { reflexology } \\
\mathrm{G} 2=\text { back } \\
\text { massage } \\
\mathrm{G} 3=\text { control } \\
\text { group }\end{array}$ & $\begin{array}{c}\text { Pittsburgh Sleep } \\
\text { Quality Index (PSQI) }\end{array}$ & $\begin{array}{c}\text { The total score of } \\
\text { PSQI improved: } \\
\text { G1 vs. G3 } \\
(P<0.05) \\
\mathrm{G} 1=11.09(3.18), \\
\mathrm{G}^{*}=5.54(2.15) \\
\mathrm{G} 3=9.20(2.42), \\
\mathrm{G}^{*}=11.88 \\
(2.47)\end{array}$ \\
\hline $\begin{array}{l}\text { Zengin et al., } \\
\text { 2018, Turkey }\end{array}$ & $\begin{array}{l}\text { Participants with } \\
\text { cancer have received } \\
\text { at least their first } \\
\text { session of } \\
\text { chemotherapy and } \\
\text { have no diagnosis of } \\
\text { sleep disorder }\end{array}$ & $\begin{array}{c}167, \mathrm{G} 1=84, \\
\mathrm{G} 2=83 \text { mean age: } \\
\mathrm{G} 1=\text { not } \\
\text { mentioned } \\
\mathrm{G} 2=\text { not } \\
\text { mentioned ( } 9 \\
\text { drop out) }\end{array}$ & $\begin{array}{c}\text { Male: } 78 \\
\text { Female: } 89\end{array}$ & $\begin{array}{l}\mathrm{G} 1=\text { foot } \\
\text { reflexology } \\
\mathrm{G} 2=\text { control } \\
\text { group }\end{array}$ & $\begin{array}{c}\text { Pittsburgh Sleep } \\
\text { Quality Index (PSQI) }\end{array}$ & $\begin{array}{c}\text { The total score of } \\
\text { PSQI improved: } \\
\text { G1 vs. G2 } \\
(P<0.001) \\
\mathrm{G} 1=12(2.7) \\
\mathrm{G} 1^{*}=5.5(2.1) \\
\mathrm{G} 2=11.3(1.9) \\
\mathrm{G} 2^{*}=13(2.4)\end{array}$ \\
\hline $\begin{array}{l}\text { Rambod et al., } \\
\text { 2019, Iran }\end{array}$ & $\begin{array}{c}\text { Patients with } \\
\text { lymphoma aged } \\
\geq 18 \text { y/o, being able to } \\
\text { speak Persian and } \\
\text { being willing to } \\
\text { participate in the } \\
\text { study }\end{array}$ & $\begin{array}{c}72, \mathrm{G} 1=36, \\
\mathrm{G} 2=36 \text { mean age: } \\
\mathrm{G} 1=41.47 \mathrm{y} / \mathrm{o} \\
(\mathrm{SD}=13.70), \\
\mathrm{G} 2=46.90 \mathrm{y} / \mathrm{o} \\
(\mathrm{SD}=15.40)\end{array}$ & $\begin{array}{l}\text { Male: } 52 \\
\text { female: } 20\end{array}$ & $\begin{array}{l}\mathrm{G} 1=\text { foot } \\
\text { reflexology } \\
\mathrm{G} 2=\text { control } \\
\text { group }\end{array}$ & $\begin{array}{c}\text { Pittsburgh Sleep } \\
\text { Quality Index (PSQI) }\end{array}$ & $\begin{array}{c}\text { The total score of } \\
\text { PSQI improved: } \\
\text { G1 vs. G2 } \\
(P<0.05) \\
\mathrm{G} 1=10.11(3.26), \\
\mathrm{G} 1^{*}=8.41(2.98) \\
\mathrm{G} 2=11.80(3.83), \\
\mathrm{G} 2^{*}=11.83 \\
(3.26)\end{array}$ \\
\hline
\end{tabular}


Table 1: Continued.

\begin{tabular}{|c|c|c|c|c|c|c|}
\hline $\begin{array}{l}\text { Authors, year, } \\
\text { country }\end{array}$ & $\begin{array}{c}\text { Main characteristics } \\
\text { of studied } \\
\text { population }\end{array}$ & $\begin{array}{c}\text { Sample } \\
\text { characteristics } \\
\text { (sample size, } \\
\text { mean age) }\end{array}$ & Sex difference & $\begin{array}{l}\text { Intervention } \\
\text { group. vs. } \\
\text { comparison } \\
\text { group }\end{array}$ & $\begin{array}{c}\text { Outcome } \\
\text { measurement tools }\end{array}$ & Outcomes \\
\hline $\begin{array}{l}\text { Malekshahi } \\
\text { et.al., 2018, Iran }\end{array}$ & $\begin{array}{l}\text { Patients between the } \\
\text { age of } 18-65 \text { y/o who } \\
\text { have sleeping } \\
\text { problems on the } \\
\text { basis of the } \\
\text { Pittsburgh } \\
\text { questionnaire, } \\
\text { undergoing } \\
\text { hemodialysis in the } \\
\text { evening and night } \\
\text { shifts }\end{array}$ & $\begin{array}{c}80, \mathrm{G} 1=40, \\
\mathrm{G} 2=40 \text { mean age: } \\
\mathrm{G} 1=\text { not } \\
\text { mentioned } \\
\mathrm{G} 2=\text { not } \\
\text { mentioned }\end{array}$ & $\begin{array}{l}\text { Male: } 53 \\
\text { female: } 27\end{array}$ & $\begin{array}{l}\mathrm{G} 1=\text { foot } \\
\text { reflexology } \\
\mathrm{G} 2=\text { control } \\
\text { group }\end{array}$ & $\begin{array}{c}\text { Pittsburgh Sleep } \\
\text { Quality Index (PSQI) }\end{array}$ & $\begin{array}{c}\text { The total score of } \\
\text { PSQI improved: } \\
\text { G1 vs. G2 } \\
(P<0.05) \\
\mathrm{G} 1=11.79(3.13), \\
\mathrm{G} 1^{*}=6.32(1.93) \\
\mathrm{G} 2=10.94(4.10), \\
\mathrm{G} 2^{*}=12.47 \\
(3.94)\end{array}$ \\
\hline $\begin{array}{l}\text { Oshvandi et al., } \\
\text { 2014, Iran }\end{array}$ & $\begin{array}{c}\text { Patients between the } \\
\text { age of } 30-80 \text { y/o who } \\
\text { have ischemic heart } \\
\text { disease hospitalized } \\
\text { in the critical care } \\
\text { unit }\end{array}$ & $\begin{array}{c}60, \mathrm{G} 1=30, \\
\mathrm{G} 2=30 \text { mean age: } \\
\mathrm{G} 1=64.17 \mathrm{y} / \mathrm{o} \\
(\mathrm{SD}=12.04), \\
\mathrm{G} 2=50.50 \mathrm{y} / \mathrm{o} \\
(\mathrm{SD}=11.40)\end{array}$ & $\begin{array}{c}\text { Male: } 34 \\
\text { Female: } 26\end{array}$ & $\begin{array}{c}\mathrm{G} 1=\text { foot } \\
\text { massage } \\
\mathrm{G} 2=\text { control } \\
\text { group }\end{array}$ & $\begin{array}{c}\text { St. Mary’s Hospital } \\
\text { Sleep Questionnaire } \\
\text { (SMHSQ) }\end{array}$ & $\begin{array}{c}\text { The total score of } \\
\text { SMHSQ } \\
\text { improved: G1 vs. } \\
\text { G2 }(P<0.05) \\
\text { G1 = } 19.67(6.25), \\
\text { G1 } 1^{*}=15.33 \\
(4.87) \mathrm{G} 2=18.93 \\
(5.87), \\
\text { G2 }{ }^{*}=18.90 \\
(5.66)\end{array}$ \\
\hline
\end{tabular}

The total score of the Verran and Synder-Halpern Sleep Scale

Patients undergoing $\quad 50, \mathrm{G} 1=25$, kidney $\quad \mathrm{G} 2=25$ mean age:

Samarehfekri et al., 2020, Iran transplantation
rrgeries suffer from surgeries suffer from postoperative pain
fatigue, and sleep disorders $\mathrm{G} 1=38.12 \mathrm{y} / \mathrm{o}$ $(\mathrm{SD}=12.87)$, $\mathrm{G} 2=38.56 \mathrm{y} / \mathrm{o}$ $(\mathrm{SD}=12)(3 \mathrm{drop}$ out)

$\begin{array}{ccc} & \text { G1 }=\text { foot } & \text { The Verran and } \\ \text { Male: } 34 & \text { massage } & \text { Snyder-Halpern } \\ \text { Female: } 16 & \text { G2 }=\text { control } & \text { Sleep Scale }\end{array}$

improved: G1 vs. G2 $(P<0.05)$

$\mathrm{G} 1=41.98$

$(\mathrm{SD}=13.92)$,

$\mathrm{G} 1^{*}=60.60$

$(\mathrm{SD}=10.75)$

$\mathrm{G} 2=42.15$

$(\mathrm{SD}=11.78)$

$\mathrm{G}^{*}=52.23$

$(\mathrm{SD}=11.76)$

The total score of RCSQ improved:

G1 vs. G2

$(P<0.05)$

$\mathrm{G} 1=430.3$

$(\mathrm{SD}=43.46)$,

$\mathrm{G}^{*}=441.8$

$(\mathrm{SD}=35.51)$

$\mathrm{G} 2=441.2$

$(\mathrm{SD}=35.18)$,

$\mathrm{G} 2 *=409.5$

Aged 18 years and $66, \mathrm{G} 1=33$, above, who are the $\mathrm{G} 2=33$ mean age:

Toygar et al., primary informal

$\mathrm{G} 1=41.52 \mathrm{y} / \mathrm{o}$

$(\mathrm{SD}=13.88)$, caregivers of cancer patients (without any $\mathrm{G} 2=39.02 \mathrm{y} / \mathrm{o}$ professional help)

$(\mathrm{SD}=12.80)$
Male: 10

Female: 56
$\mathrm{G} 1=$ foot reflexology $\mathrm{G} 2=$ control group (shame intervention)
Richard-Campbell Sleep Questionnaire (RCSQ) state-trait anxiety inventory

(STAI)
$(\mathrm{SD}=50.08)$ the total score of

STAI improved:

$$
\begin{gathered}
\mathrm{G} 1 \text { vs. } \mathrm{G} 2 \\
(P<0.05) \\
\mathrm{G} 1=46.67 \\
(\mathrm{SD}=7.21) \\
\mathrm{G} 1^{*}=38.91 \\
(\mathrm{SD}=5.63) \\
\mathrm{G} 2=47.94 \\
(\mathrm{SD}=10.62), \\
\mathrm{G} 2^{*}=46.30 \\
(\mathrm{SD}=11.29)
\end{gathered}
$$


TABle 1: Continued.

\begin{tabular}{|c|c|c|c|c|c|c|}
\hline $\begin{array}{l}\text { Authors, year, } \\
\text { country }\end{array}$ & $\begin{array}{c}\text { Main characteristics } \\
\text { of studied } \\
\text { population }\end{array}$ & $\begin{array}{c}\text { Sample } \\
\text { characteristics } \\
\text { (sample size, } \\
\text { mean age) }\end{array}$ & Sex difference & $\begin{array}{l}\text { Intervention } \\
\text { group. vs. } \\
\text { comparison } \\
\text { group }\end{array}$ & $\begin{array}{c}\text { Outcome } \\
\text { measurement tools }\end{array}$ & Outcomes \\
\hline $\begin{array}{l}\text { Bahrami et al., } \\
\text { 2019, Iran }\end{array}$ & $\begin{array}{l}\text { A female patient } \\
\text { aged } \geq 60 \text { y/o } \\
\text { diagnosed with acute } \\
\text { coronary syndrome } \\
\text { consisting of angina } \\
\text { pectoris and } \\
\text { myocardia } \\
\text { infraction, no } \\
\text { anxiolytics and } \\
\text { sedative medications } \\
\text { in the last four hours } \\
\text { before the } \\
\text { intervention }\end{array}$ & $\begin{array}{c}90, \mathrm{G} 1=45, \\
\mathrm{G} 2=45 \text { mean age: } \\
\mathrm{G} 1=72.86 \mathrm{y} / \mathrm{o} \\
(\mathrm{SD}=7.98) \\
\mathrm{G} 2=72.62 \mathrm{y} / \mathrm{o} \\
(\mathrm{SD}=7.93)\end{array}$ & $\begin{array}{l}\text { Male: } 0 \\
\text { Female: } 90\end{array}$ & $\begin{array}{c}\mathrm{G} 1=\text { foot } \\
\text { reflexology } \\
\mathrm{G} 2=\text { control } \\
\text { group }\end{array}$ & $\begin{array}{c}\text { Hospital depression } \\
\text { scale (HADS-D) } \\
\text { hospital anxiety scale } \\
(\text { HADS-A) }\end{array}$ & $\begin{array}{c}\text { The total score of } \\
\text { HADS-D } \\
\text { improved: G1 vs. } \\
\text { G2 }(P<0.05) \\
\mathrm{G} 1=13.66 \\
(\mathrm{SD}=4.64), \\
\mathrm{G} 1^{*}=8.42 \\
(\mathrm{SD}=3.62) \\
\mathrm{G} 2=11.74 \\
(\mathrm{SD}=4.29), \\
\mathrm{G} 2^{*}=11.11 \\
(\mathrm{SD}=3.42) \text { the } \\
\text { total score of } \\
\mathrm{HADS}-\mathrm{A} \\
\text { improved: } \mathrm{G} 1 \text { vs. } \\
\mathrm{G} 2(P<0.05) \\
\mathrm{G} 1=13.77 \\
(\mathrm{SD}=4.39), \\
\mathrm{G} 1^{*}=8.53 \\
(\mathrm{SD}=3.71) \\
\mathrm{G} 2=11.66 \\
(\mathrm{SD}=4.24), \\
\mathrm{G} 2^{*}=11.06 \\
(\mathrm{SD}=3.19)\end{array}$ \\
\hline
\end{tabular}

The total score of HADS-D

improved: G1 vs. G2 $(P<0.01)$

$\mathrm{G} 1=9.31$

$(\mathrm{SD}=4.47)$,

$\mathrm{G}^{*}=8.03$

Gynaecologic cancer patients receiving chemotherapy and

Noh et al., 2019, hospitalized in the South Korea gynaecological ward, who received shortterm chemotherapy

(at least 2 weeks chemotherapy)
$63, \mathrm{G} 1=32$,

$\mathrm{G} 2=31$ mean age:

$\mathrm{G} 1=56.34 \mathrm{y} / \mathrm{o}$

$(\mathrm{SD}=9.04)$,

$\mathrm{G} 2=55.36 \mathrm{y} / \mathrm{o}$

$(\mathrm{SD}=9.96)(1$

drop out)
Male: 0

Female: 63
$\mathrm{G} 1$ = self-foot reflexology
$\mathrm{G} 2=$ control group

Hospital depression scale (HADS-D);

hospital anxiety scale

(HADS-A)
$(\mathrm{SD}=4.28)$

$\mathrm{G} 2=8.58$

$(\mathrm{SD}=4.36)$,

$\mathrm{G}^{*}=9.48$

$(\mathrm{SD}=4.14)$ the

total score of

HADS-A

improved: G1 vs.

G2 $(P<0.01)$

$\mathrm{G} 1=7.25$

$(\mathrm{SD}=4.05)$,

$\mathrm{Gl}^{*}=5.69$

$(\mathrm{SD}=3.46)$

$\mathrm{G} 2=6.48$

( $\mathrm{SD}=3.06)$,

$\mathrm{G}^{*}=7.39$

$(\mathrm{SD}=3.23)$ 
TABle 1: Continued.

\begin{tabular}{|c|c|c|c|c|c|c|}
\hline $\begin{array}{l}\text { Authors, year, } \\
\text { country }\end{array}$ & $\begin{array}{c}\text { Main characteristics } \\
\text { of studied } \\
\text { population }\end{array}$ & $\begin{array}{c}\text { Sample } \\
\text { characteristics } \\
\text { (sample size, } \\
\text { mean age) }\end{array}$ & Sex difference & $\begin{array}{l}\text { Intervention } \\
\text { group. vs. } \\
\text { comparison } \\
\text { group }\end{array}$ & $\begin{array}{c}\text { Outcome } \\
\text { measurement tools }\end{array}$ & Outcomes \\
\hline $\begin{array}{l}\text { Mahdavipour } \\
\text { et al., 2019, Iran }\end{array}$ & $\begin{array}{l}\text { Women during their } \\
\text { menopausal period, } \\
\text { aged } 40-60 \text { y/o, } \\
\text { diagnosis of } \\
\text { depression by a } \\
\text { psychiatrist based on } \\
\text { DSM-IV, and the } \\
\text { total depression } \\
\text { score }>14 \text { based on } \\
\text { the Beck Depression } \\
\text { Inventory }\end{array}$ & $\begin{array}{c}90, \mathrm{G} 1=45, \\
\mathrm{G} 2=45 \text { mean age: } \\
\mathrm{G} 1=54.18 \text { y/o } \\
(\mathrm{SD}=3.90) \\
\mathrm{G} 2=52.23 \mathrm{y} / \mathrm{o} \\
(\mathrm{SD}=11.6)(10 \\
\text { drop out })\end{array}$ & $\begin{array}{c}\text { Male: } 0 \\
\text { Female: } 90\end{array}$ & $\begin{array}{c}\mathrm{G} 1=\text { foot } \\
\text { reflexology } \\
\mathrm{G} 2=\text { control } \\
\text { group }\end{array}$ & $\begin{array}{l}\text { Beck Depression } \\
\text { Inventory-second } \\
\text { edition (BDI-II) }\end{array}$ & $\begin{array}{c}\text { The total score of } \\
\text { BDI-II improved: } \\
\text { G1 vs. G2 } \\
(P<0.001) \\
\mathrm{G} 1=26.97 \\
(\mathrm{SD}=4.47), \\
\mathrm{G} 1^{*}=22.55 \\
(\mathrm{SD}=5.18) \\
\mathrm{G} 2=26.15 \\
(\mathrm{SD}=5.01), \\
\mathrm{G} 2^{*}=26.22 \\
(\mathrm{SD}=5.14)\end{array}$ \\
\hline
\end{tabular}

The total score of

DASS-21

depression

improved: G1 vs.

G3 $(P=0.03)$

$\mathrm{G} 1=20.72$

$(\mathrm{SD}=7.56)$,

$\mathrm{G}^{*}=13.20$

$75, \mathrm{G} 1=25$,

$\mathrm{G} 2=25, \mathrm{G} 3=25$

Female patients aged

$18-75 \mathrm{y} / \mathrm{o}$, with a

Soheili et al., 2017, Iran multiple sclerosis by a medicine specialist

mean age:

$\mathrm{G} 1=34.4 \mathrm{y} / \mathrm{o}$

$(\mathrm{SD}=6.6)$,

$\mathrm{G} 2=33.9 \mathrm{y} / \mathrm{o}$

$(\mathrm{SD}=5.6)$

$\mathrm{G} 3=34.0 \mathrm{y} / \mathrm{o}$

$(\mathrm{SD}=7.7)$
$\mathrm{G} 1=$ foot

Male: 0

Female: 75

reflexology

$\mathrm{G} 2$ = relaxation

$\mathrm{G} 3=$ control

group
Depression, anxiety

and stress scale- 21

(DASS-21)
$(\mathrm{SD}=6.16)$

$\mathrm{G} 3=19.52$

$(\mathrm{SD}=6.06)$,

$\mathrm{G}^{*}=18.64$

$(\mathrm{SD}=6.99)$ the

total score of

DASS-21 anxiety

improved: G1 vs.

G3 $(P=0.03)$

$\mathrm{G} 1=16.72$

$(\mathrm{SD}=6.66)$,

$\mathrm{G} 1^{*}=10.40$

$(\mathrm{SD}=7.37)$

$\mathrm{G} 3=16.80$

$(\mathrm{SD}=6.90)$,

$\mathrm{G}^{*}=14.88$

$(\mathrm{SD}=6.50)$

The total score of

STAI improved:

G1 vs. G2

$(P=0.0001)$

$\mathrm{G} 1=53.24$

$(\mathrm{SD}=4.29)$,

$\mathrm{G1}^{*}=45.24$

$(\mathrm{SD}=3.32)$

$\mathrm{G} 2=49.62$

$(\mathrm{SD}=5.31)$,

$\mathrm{G}^{*}=43.70$

$(\mathrm{SD}=5.06)$ 
TABle 1: Continued.

\begin{tabular}{|c|c|c|c|c|c|c|}
\hline $\begin{array}{l}\text { Authors, year, } \\
\text { country }\end{array}$ & $\begin{array}{c}\text { Main characteristics } \\
\text { of studied } \\
\text { population }\end{array}$ & $\begin{array}{c}\text { Sample } \\
\text { characteristics } \\
\text { (sample size, } \\
\text { mean age) }\end{array}$ & Sex difference & $\begin{array}{l}\text { Intervention } \\
\text { group. vs. } \\
\text { comparison } \\
\text { group }\end{array}$ & $\begin{array}{c}\text { Outcome } \\
\text { measurement tools }\end{array}$ & Outcomes \\
\hline
\end{tabular}

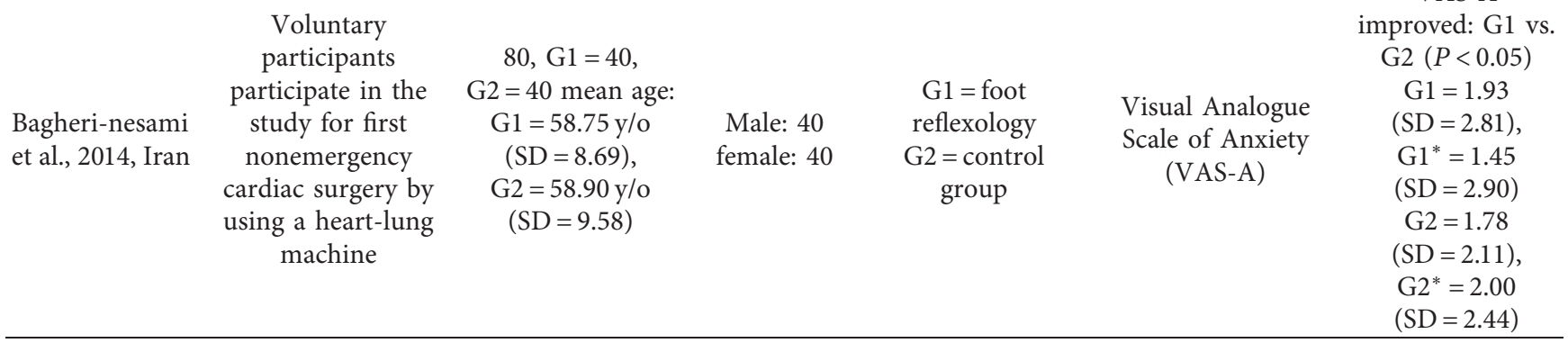

Participants aged $\geq 18$ y/o, candidate for coronary angiography in

Khaledifar hospital, absence of et al., 2017, Iran acute psychological disorders, or use of antistress drugs within recent 48 hours
$75, \mathrm{G} 1=25$,

$\mathrm{G} 2=25, \mathrm{G} 3=25$

mean age:

$\mathrm{G} 1=67.2 \mathrm{y} / \mathrm{o}$

$(\mathrm{SD}=11.8)$,

$\mathrm{G} 2=67.0 \mathrm{y} / \mathrm{o}$

$(\mathrm{SD}=11.1)$

$\mathrm{G} 3=64.7 \mathrm{y} / \mathrm{o}$

$(\mathrm{SD}=12.1)$

The total score of

STAI improved:

G1 vs. G3

$(P<0.05)$

$\mathrm{G} 1=60.60$

Male: 38 reflexology

female: 37

G2 = massage

therapy

$\mathrm{G} 3$ = control

group
State-Trait Anxiety

Inventory (STAI)

$(\mathrm{SD}=7.20)$,

$\mathrm{G}^{*}=34.70$

$(\mathrm{SD}=4.70)$

$\mathrm{G} 3=47.80$

$(\mathrm{SD}=9.60)$,

$\mathrm{G}^{*}=46.50$

$(\mathrm{SD}=9.20)$

\section{Female, aged} 20-35 y/o, being primiparous, giving birth to a living and

Saatsaz et al., 2016, Iran healthy child, being conscious, and having junior high school or higher degree of education to comprehend the numerical pain scale
$106, \mathrm{G} 1=52$,

mean age:

$\mathrm{G} 1=27.04 \mathrm{y} / \mathrm{o}$

$(\mathrm{SD}=2.77)$,

$\mathrm{G} 2=26.73 \mathrm{y} / \mathrm{o}$

$(\mathrm{SD}=3.81)$,

$\mathrm{G} 3=27.75 \mathrm{y} / \mathrm{o}$

$(\mathrm{SD}=3.22)$
$\mathrm{G} 2=52, \mathrm{G} 3=52$
Male: 0

Female: 106

$$
\text { massage }
$$

$\mathrm{G} 1=$ foot

$\mathrm{G} 2$ = foot and

hand massage

$\mathrm{G} 3=$ control

group
State-Trait Anxiety

Inventory (STAI)
The total score of

STAI improved:

G1 vs. G3

$(P<0.05)$

$\mathrm{G} 1=31.52$

$(\mathrm{SD}=9.93)$,

$\mathrm{Gl}^{*}=28.23$

$(\mathrm{SD}=8.88)$

$\mathrm{G} 3=30.17$

$(\mathrm{SD}=6.98)$,

$\mathrm{G}^{*}=30.38$

$(\mathrm{SD}=6.93)$

The total score of

Patients who had undergone tibial shaft fracture surgery; aged $\geq 18 \mathrm{y} /$

Pasyar et al., o; an open reduction 2018, Iran and internal fixation surgery for a tibial fracture, hospital admission for at least 1 day after surgery
$66, \mathrm{G} 1=33$,

$\mathrm{G} 2=33 \mathrm{G} 1=$ not mentioned $\mathrm{G} 2=$ not mentioned
Male: 53

female: 13
$\mathrm{G} 1=$ foot reflexology $\mathrm{G} 2$ = control group
STAI improved:

G1 vs. G2

$(P<0.05)$

$\mathrm{G} 1=54.72$

$(\mathrm{SD}=7.36)$,

$\mathrm{G}^{*}=42.84$

$(\mathrm{SD}=6.50)$

$\mathrm{G} 2=57.48$

$(\mathrm{SD}=9.14)$,

$\mathrm{G}^{*}=58.36$

$(\mathrm{SD}=10.37)$ 
TABle 1: Continued.

\begin{tabular}{|c|c|c|c|c|c|c|}
\hline $\begin{array}{l}\text { Authors, year, } \\
\text { country }\end{array}$ & $\begin{array}{c}\text { Main characteristics } \\
\text { of studied } \\
\text { population }\end{array}$ & $\begin{array}{c}\text { Sample } \\
\text { characteristics } \\
\text { (sample size, } \\
\text { mean age) }\end{array}$ & Sex difference & $\begin{array}{l}\text { Intervention } \\
\text { group. vs. } \\
\text { comparison } \\
\text { group }\end{array}$ & $\begin{array}{c}\text { Outcome } \\
\text { measurement tools }\end{array}$ & Outcomes \\
\hline $\begin{array}{l}\text { Koras et al., } \\
\text { 2019, Turkey }\end{array}$ & $\begin{array}{l}\text { Patients age } \geq 18 \text { y/o } \\
\text { who underwent } \\
\text { laparoscopic } \\
\text { cholecystectomy } \\
\text { without any } \\
\text { complication with } \\
\text { pain severity greater } \\
\text { than } 4 \text { on VAS } \\
\text { (visual analogue } \\
\text { scale) after surgery }\end{array}$ & $\begin{array}{c}167, \mathrm{G} 1=85, \\
\mathrm{G} 2=82 \text { mean age: } \\
\mathrm{G} 1=\text { not } \\
\text { mentioned } \\
\mathrm{G} 2=\text { not } \\
\text { mentioned }\end{array}$ & $\begin{array}{c}\text { Male: } 50 \\
\text { female: } 117\end{array}$ & $\begin{array}{c}\mathrm{G} 1=\text { foot } \\
\text { massage } \\
\mathrm{G} 2=\text { control } \\
\text { group }\end{array}$ & $\begin{array}{c}\text { State-Trait Anxiety } \\
\text { Inventory (STAI) }\end{array}$ & $\begin{array}{c}\text { The total score of } \\
\text { STAI improved: } \\
\text { G1 vs. G2 } \\
(P<0.05) \\
\mathrm{G} 1=49.74 \\
(\mathrm{SD}=13.54) \\
\mathrm{G}^{*}=28.67 \\
(\mathrm{SD}=9.12) \\
\mathrm{G} 2=43.67 \\
(\mathrm{SD}=8.11) \\
\mathrm{G} 2^{*}=51.84 \\
(\mathrm{SD}=6.61)\end{array}$ \\
\hline
\end{tabular}

The total score of

STAI improved:

Men and women $55, \mathrm{G} 1=27$, aged 20 to 70 who lived in or near

Eguchi et al., 2016, Japan

Matsuyama, Ehime Prefecture, Japan $\mathrm{G} 2=28$ mean age: $\mathrm{G} 1=49.0 \mathrm{y} / \mathrm{o}$ $(\mathrm{SD}=13.6)$, $\mathrm{G} 2=48.8 \mathrm{y} / \mathrm{o}$ $(\mathrm{SD}=11.4)$ female: 50

reflexology

$\mathrm{G} 2$ = control

group

G1 vs. G2

$(P<0.05)$

$\mathrm{G} 1=41.1$

$(\mathrm{SD}=11.2)$,

$\mathrm{G1}^{*}=38.0$

$(\mathrm{SD}=9.4)$

$\mathrm{G} 2=40.6$

$(\mathrm{SD}=10.0)$,

$\mathrm{G}^{*}=40.0$

$(\mathrm{SD}=9.2)$

The total score of

Voluntary participants who have undergone abdominal

Ozturk et al., 2018, Turkey hysterectomy operation and reported

postoperation pain of 3 or above

according to visual analog scale

STAI improved:

G1 vs. G2

$(P<0.05)$

$\mathrm{G} 1=58.87$

G2 $=31$ mean age: Male: 0

$47.23 \mathrm{y} / \mathrm{o}$

$(\mathrm{SD}=4.71)$ female: 63
$\mathrm{G} 1=$ foot

reflexology

$\mathrm{G} 2$ = control group
State-Trait Anxiety

Inventory (STAI)

$(\mathrm{SD}=4.81)$,

$\mathrm{G}^{*}=45.75$

$(\mathrm{SD}=4.25)$

$\mathrm{G} 2=57.32$

$(\mathrm{SD}=4.81)$,

$\mathrm{G} 2 *=55.96$

$(\mathrm{SD}=3.85)$

The total score of

STAI improved:

undergoing coronary

angiography, aged

40-80 y/o, had

neither health

$150, \mathrm{G} 1=50$,

problems nor arterial $\mathrm{G} 2=50, \mathrm{G} 3=50$

line in the feet, received no anxiolytic agent during the past

48 hours before the intervention
G1 vs. G3

$(P<0.05)$

$\mathrm{G} 1=61.68$

Male: 150

$\mathrm{G} 1=$ foot

reflexology

$\mathrm{G} 2$ = placebo

State-Trait Anxiety group group

$\mathrm{G} 3=$ control

Inventory (STAI)

$$
(\mathrm{SD}=4.6)
$$

Female: 0
$(\mathrm{SD}=-)$,

$\mathrm{G}^{*}=45.58$

$(\mathrm{SD}=-)$

$\mathrm{G} 2=60.52$

$(\mathrm{SD}=-)$,

$\mathrm{G}^{*}=59.14$

$(\mathrm{SD}=-)$ 
TABle 1: Continued.

\begin{tabular}{|c|c|c|c|c|c|c|}
\hline $\begin{array}{l}\text { Authors, year, } \\
\text { country }\end{array}$ & $\begin{array}{c}\text { Main characteristics } \\
\text { of studied } \\
\text { population }\end{array}$ & $\begin{array}{c}\text { Sample } \\
\text { characteristics } \\
\text { (sample size, } \\
\text { mean age) }\end{array}$ & Sex difference & $\begin{array}{l}\text { Intervention } \\
\text { group. vs. } \\
\text { comparison } \\
\text { group }\end{array}$ & $\begin{array}{c}\text { Outcome } \\
\text { measurement tools }\end{array}$ & Outcomes \\
\hline $\begin{array}{l}\text { Shahsavari } \\
\text { et al., 2017, Iran }\end{array}$ & $\begin{array}{l}\text { Patients between the } \\
\text { age of } 18-60 \mathrm{y} / \mathrm{o} \text {, no } \\
\text { lesion or disorder on } \\
\text { the feet and other } \\
\text { conditions affecting } \\
\text { the feet, no previous } \\
\text { history of } \\
\text { bronchoscopy, or } \\
\text { participation in } \\
\text { similar studies }\end{array}$ & $\begin{array}{c}80, \mathrm{G} 1=40, \\
\mathrm{G} 2=40 \text { mean age: } \\
\mathrm{G} 1=45.55 \mathrm{y} / \mathrm{o} \\
(\mathrm{SD}=1.78) \\
\mathrm{G} 2=48.23 \mathrm{y} / \mathrm{o} \\
(\mathrm{SD}=1.72)\end{array}$ & $\begin{array}{c}\text { Male: } 41 \\
\text { female: } 39\end{array}$ & $\begin{array}{c}\mathrm{G} 1=\text { foot } \\
\text { reflexology } \\
\mathrm{G} 2=\text { control } \\
\text { group }\end{array}$ & $\begin{array}{l}\text { Visual Analogue } \\
\text { Scale of Anxiety } \\
\text { (VAS-A) }\end{array}$ & $\begin{array}{c}\text { The total score of } \\
\text { VAS-A } \\
\text { improved: } \mathrm{G} 1 \text { vs. } \\
\text { G2 }(P<0.05) \\
\mathrm{G} 1=4.35 \\
(\mathrm{SD}=2.08) \\
\mathrm{G} 1^{*}=2.83 \\
(\mathrm{SD}=1.45) \\
\mathrm{G} 2=3.78 \\
(\mathrm{SD}=1.83) \\
\mathrm{G} 2^{*}=4.88 \\
(\mathrm{SD}=2.15)\end{array}$ \\
\hline
\end{tabular}

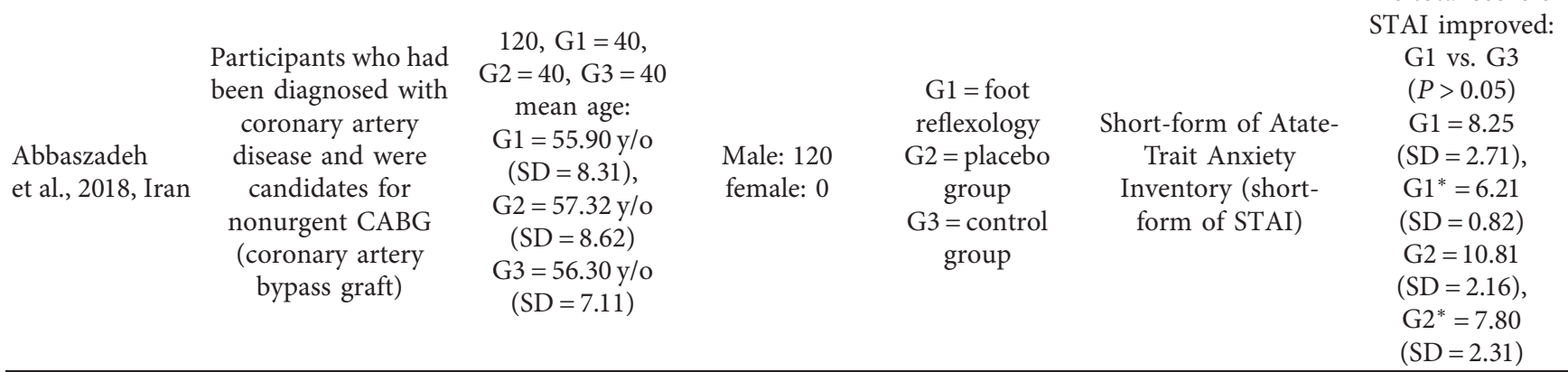

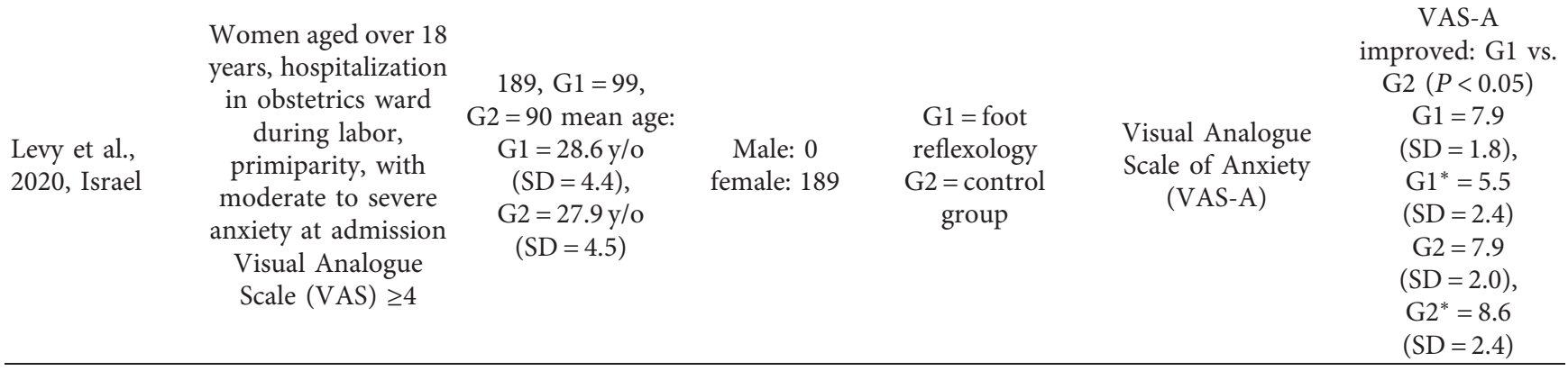

BDI-II = Beck Depression Inventory-second edition; $\mathrm{CABG}=$ coronary artery bypass graft; DASS-21 = depression, anxiety, and stress scale-21; DSMIV = Diagnostic and Statistical Manual of mental disorders, $4^{\text {th }}$ edition; G1 = group 1, G2 = group 2, G3 = group 3; HADS-A= hospital anxiety and depression scale-anxiety; HADS-D = hospital anxiety and depression scale-depression; PSQI = Pittsburgh Sleep Quality Index; SMHSQ = St. Mary's Hospital Sleep Questionnaire; STAI = State-Trait Anxiety Inventory; RCSQ = Richard-Campbell Sleep Questionnaire; VAS-A = visual analogue scale for anxiety.

3.4. Efficacy Analysis (Results from Each Meta-Analysis) Primary Outcomes. The sizes of effect for selected studies were prominent in depression, anxiety, and sleep disturbance. The data revealed in Table 4 that foot reflexology intervention resulted in significant improvement in adults with depression, anxiety, and sleep problems.

3.4.1. Depression. Four studies $[7,50,54,55]$ investigated depression as the primary outcome following foot reflexology intervention by using different depression outcome measurement tools. These tools included the Beck Depression Inventory Scale; hospital anxiety and depression scale; and depression, anxiety, and stress scale-21 and were used in our meta-analysis. Hedges' $g$ for the overall effect size was -0.921 , and the $95 \%$ CI was -1.246 to -0.595 (Figure 3). The sample collection sizes of effect for sample collection all came out negative, with Hedges' $g$ ranging from -0.511 to -1.298 . Reviewing the results, it is clear that there was significant reduction in depression following foot reflexology intervention, with a large effect size. There was mean heterogeneity among the studies of depression $\left(Q=5.42, P=0.143, I^{2}=44.74\right)$.

3.4.2. Anxiety. The sixteen studies $[45-48,50,52-62]$ examined anxiety as the primary outcome following foot reflexology intervention by using different anxiety outcome measurement tools such as the hospital anxiety and depression scale; depression, anxiety, and stress scale-21; State- 
Trait Anxiety Inventory; and Visual Analogue Scale of Anxiety and were included in our meta-analysis. Hedges' $g$ for the overall effect size was -1.237 , and the $95 \%$ CI was -1.682 to -0.791 (Figure 4 ). The sample collection effect sizes all came out negative, with Hedges' $g$ ranging from -0.259 to -3.644 . These results suggested that the overall reduction in anxiety following foot reflexology intervention was significant, with a large effect size. Heterogeneity among the studies of anxiety was considerably large $(Q=217.41$, $\left.P<0.001, I^{2}=93.10\right)$.

3.4.3. Sleep Quality. Hedges' $g$ of the ten studies $[8,41-44,49,51,62-64]$ examined sleep quality following foot reflexology intervention by using different outcome measurement tools such as the Pittsburgh Sleep Quailty Index; the Verran and Snyder-Halpern Sleep Scale; St. Mary's Hospital Sleep Questionnaire; and Richard-Campbell Sleep Questionnaire which were included in our metaanalysis. Hedges' $g$ for the overall effect size was -1.665 , and the $95 \%$ CI was -2.361 to -0.970 (Figure 5). The effect sizes for sample collection were unsurprisingly all negative, with Hedges' $g$ ranging from -0.548 to -3.621 . The meta-analysis revealed that the overall improvement in sleep quality following foot reflexology intervention was significant, with a large effect size. Considerable heterogeneity was observed among the studies in sleep quality where the outcomes measured $\left(Q=144.87, P<0.001, I^{2}=93.78\right)$.

Substantial heterogeneity was found in the anxiety and sleep quality studies. Therefore, subgroup analyses along with moderator and metaregression analyses were conducted to further explore the determinations of the heterogeneity.

3.5. Secondary Outcomes (Safety). No adverse events were reported in the few RCTs on foot reflexology intervention for depression, anxiety, and sleep quality. Most of the included studies failed to report this aspect. Dropouts were not treated as adverse events not only because they were not explicitly explaining their personal reasons for dropout in the original study but also because our research material lacked subject commentary.

3.6. Subgroup Analyses and Metaregression Analyses of Anxiety and Sleep Quality. Subgroup analyses and metaregression analyses to investigate any possible confounding clinical variables within the studies are presented in Table 5.

3.6.1. Anxiety. Four RCTs revealed evidence for the effects of foot reflexology when compared with the control group in reducing the anxiety level before adult undergoing coronary angiography (Hedges' $g=-1.426$, 95\% CI was -2.278 to $-0.575, P<0.001)$. Two RCTs revealed evidence for the effects of foot reflexology compared with the control group in reducing the anxiety level for delivering women (Hedges' $g=-0.869,95 \%$ CI was -1.702 to $-0.869, P=0.041)$. Significant subgroup differences were identified for the outcome measures (STAI vs. Others; Hedges' $g=-1.534$ vs.
$-0.894, P<0.001)$. Our subgroup analysis performed one session of foot reflexology intervention, before or after interventional surgery, which would be more effective than numerous sessions of foot reflexology intervention, as according to other interventional surgeries or procedure studies (one session vs. numerous sessions; Hedges' $g=-1.553$ vs. $-0.849, P<0.001)$. Other subgroup analysis indicated cardiovascular surgery or an interventional procedure was less effective than other surgery or interventional procedures (cardiovascular vs. other surgery; Hedges' $g=-1.060$ vs. $-2.340, P<0.001$ ), which significantly reduced the anxiety level of psychological symptoms. The selection bias including random sequence generation and allocation concealment of study also showed significant differences in interactions between subgroups $(P<0.05)$.

In the exploratory metaregression analysis of anxiety, no significant relationship was observed between the effect size for mean age $(P=0.852)$ and total length of intervention in one time period $(P=0.903)$.

3.6.2. Sleep Quality. Subgroup analysis was performed using the parameters study group and participants type. However, results of the subgroup analysis indicated that heterogeneity may have resulted from the abovementioned factors. While performing the metaregression, the mean age of participants, duration of intervention sessions, and total foot reflexology intervention time were required as possible moderating variables. The selection bias including random sequence generation and allocation concealment of study also showed significant differences in interactions between subgroups $(P<0.05)$.

Regression analyses revealed a positive correlation with the total length of foot reflexology intervention time $(P=0.002)$ and duration of intervention sessions $(P=0.01)$, indicating that the more the total length of foot reflexology intervention time and duration of intervention sessions, the more likely it is to have significant results. However, the mean age of participants did not report any significant impact $(P=0.897)$.

\section{Discussion}

4.1. Summary of Evidence. We analyzed the impact on foot reflexology on depression, anxiety, and sleep quality. Metaanalysis for improvement of psychological symptoms indicated that the foot reflexology could effectively relieve depression, anxiety, and sleep quality. However, effect sizes of various studies were heterogeneous. In addition, not only did we focus on the possible moderating clinical factors but also investigated the possible confounding effect by the different measurement tools.

Overall, the application of foot reflexology was not associated with degradation of psychological symptoms or a rapid increase in adverse effects. Only a few studies explicitly assessed safety-related, nonserious adverse events. Foot reflexology is most likely a comparatively safe practice for this population. However, future RCTs should take more measures to establish even more accurate 
TABLE 2: Characteristics of foot reflexology programs and outcomes assessment of studies included in meta-analysis.

\begin{tabular}{|c|c|c|c|c|c|c|c|}
\hline Authors, year & $\begin{array}{c}\text { Frequency } \\
\text { (sessions/ } \\
\text { week) } \\
\end{array}$ & $\begin{array}{l}\text { Session length } \\
\text { (mins/session) }\end{array}$ & $\begin{array}{c}\text { Duration } \\
\text { (weeks/ } \\
\text { study) } \\
\end{array}$ & $\begin{array}{c}\text { Number of } \\
\text { sessions/study } \\
\text { total length/study }\end{array}$ & $\begin{array}{l}\text { Safety } \\
\text { (adverse } \\
\text { events) } \\
\end{array}$ & $\begin{array}{l}\text { Lasting effects } \\
\text { and duration }\end{array}$ & $\begin{array}{l}\text { Adherence } \\
\text { rate }(\%)\end{array}$ \\
\hline $\begin{array}{l}\text { Valizadeh et al., } \\
2015\end{array}$ & 1 & $\begin{array}{l}20 \text { (total } 20 \mathrm{~min}, \\
10 \mathrm{~min} \text { for each } \\
\text { foot) }\end{array}$ & 6 & 6 (2 hours) & $\begin{array}{l}\text { Not } \\
\text { reported }\end{array}$ & Not reported & $23 / 23=100 \%$ \\
\hline Li et al., 2011 & 5 & $\begin{array}{l}30 \text { (total } 30 \mathrm{~min} \text {, } \\
15 \mathrm{~min} \text { for each } \\
\text { foot) }\end{array}$ & 1 & 5 (2.5 hours) & $\begin{array}{l}\text { Not } \\
\text { reported }\end{array}$ & Not reported & $32 / 34=94 \%$ \\
\hline Bakir et al., 2018 & 1 & $\begin{array}{l}60 \text { (total } 60 \mathrm{~min} \text {, } \\
30 \mathrm{~min} \text { for each } \\
\text { foot) }\end{array}$ & 6 & 6 (6 hours) & $\begin{array}{l}\text { Not } \\
\text { reported }\end{array}$ & Not reported & $30 / 31=96 \%$ \\
\hline Unal et al., 2016 & 2 & $\begin{array}{l}30 \text { (total } 30 \mathrm{~min} \text {, } \\
15 \mathrm{~min} \text { for each } \\
\text { foot) }\end{array}$ & 4 & 8 (4 hours) & $\begin{array}{l}\text { Not } \\
\text { reported }\end{array}$ & Not reported & $35 / 35=100 \%$ \\
\hline Zengin et al., 2018 & 2 & $\begin{array}{l}30 \text { (total } 30 \mathrm{~min} \text {, } \\
15 \mathrm{~min} \text { for each } \\
\text { foot) }\end{array}$ & 8 & 16 (8 hours) & $\begin{array}{l}\text { Not } \\
\text { reported }\end{array}$ & Not reported & $84 / 88=95 \%$ \\
\hline $\begin{array}{l}\text { Rambod et al., } \\
2019\end{array}$ & 5 & $\begin{array}{l}30 \text { (total } 30 \mathrm{~min} \text {, } \\
15 \mathrm{~min} \text { for each } \\
\text { foot) }\end{array}$ & 1 & 5 (2.5 hours) & $\begin{array}{l}\text { No side } \\
\text { effect }\end{array}$ & Not reported & $36 / 36=100 \%$ \\
\hline $\begin{array}{l}\text { Malekshahi et al., } \\
2018\end{array}$ & 3 & $\begin{array}{l}10 \text { (totally } \\
10 \mathrm{~min}, 5 \mathrm{~min} \text { for } \\
\text { each foot) }\end{array}$ & 4 & 12 (2 hours) & $\begin{array}{l}\text { Not } \\
\text { reported }\end{array}$ & Not reported & $40 / 40=100 \%$ \\
\hline $\begin{array}{l}\text { Oshvandi et al., } \\
2014\end{array}$ & 2 & $\begin{array}{l}20 \mathrm{~min} \text { (totally } \\
20 \mathrm{~min}, 10 \mathrm{~min} \\
\text { for each foot) }\end{array}$ & 1 & 2 (0.66 hours) & $\begin{array}{l}\text { Not } \\
\text { reported }\end{array}$ & Not reported & $30 / 30=100 \%$ \\
\hline $\begin{array}{l}\text { Samarehfekri } \\
\text { et al., } 2020\end{array}$ & 3 & $\begin{array}{l}30 \mathrm{~min} \text { (totally } \\
30 \mathrm{~min}, 15 \mathrm{~min} \\
\text { for each foot) }\end{array}$ & 1 & 3 (1.5 hours) & $\begin{array}{l}\text { No side } \\
\text { effect }\end{array}$ & $\begin{array}{l}1 \text { week after } \\
\text { intervention }\end{array}$ & $25 / 26=96 \%$ \\
\hline Toygar et al., 2020 & 3 & $\begin{array}{l}30 \mathrm{~min} \text { (totally } \\
30 \mathrm{~min}, 15 \mathrm{~min} \\
\text { for each foot) }\end{array}$ & 1 & 3 (1.5 hours) & $\begin{array}{l}\text { Not } \\
\text { reported }\end{array}$ & Not reported & $33 / 33=100 \%$ \\
\hline $\begin{array}{l}\text { Bahrami et al., } \\
2019\end{array}$ & 1 & $\begin{array}{l}20 \mathrm{~min} \text { (totally } \\
20 \mathrm{~min}, 10 \mathrm{~min} \\
\text { for each foot) }\end{array}$ & 1 & 1 (0.33 hours) & $\begin{array}{l}\text { No side } \\
\text { effect }\end{array}$ & Not reported & $45 / 45=100 \%$ \\
\hline Noh et al., 2019 & 3 & $\begin{array}{l}30 \mathrm{~min} \text { (totally } \\
30 \mathrm{~min}, 15 \mathrm{~min} \\
\text { for each foot) }\end{array}$ & 6 & 18 (9 hours) & $\begin{array}{l}\text { Not } \\
\text { reported }\end{array}$ & Not reported & $32 / 33=96 \%$ \\
\hline $\begin{array}{l}\text { Mahdavipour } \\
\text { et al., } 2019\end{array}$ & 2 & $\begin{array}{l}30 \mathrm{~min} \text { (totally } \\
30 \mathrm{~min}, 15 \mathrm{~min} \\
\text { for each foot) }\end{array}$ & 6 & 12 (6 hours) & $\begin{array}{l}\text { Not } \\
\text { reported }\end{array}$ & $\begin{array}{l}2 \text { months after } \\
\text { intervention }\end{array}$ & $45 / 50=90 \%$ \\
\hline Soheili et al., 2017 & 2 & $\begin{array}{l}40 \mathrm{~min} \text { (totally } \\
40 \mathrm{~min}, 20 \mathrm{~min} \\
\text { for each foot) }\end{array}$ & 4 & 8 (5.33 hours) & $\begin{array}{c}\text { Not } \\
\text { reported }\end{array}$ & Not reported & $25 / 25=100 \%$ \\
\hline $\begin{array}{l}\text { Vardanjani et al., } \\
2013\end{array}$ & 1 & $30 \mathrm{~min}$ & 1 & 1 (0.5 hours) & $\begin{array}{l}\text { Not } \\
\text { reported }\end{array}$ & Not reported & $50 / 50=100 \%$ \\
\hline $\begin{array}{l}\text { Bagheri-nesami } \\
\text { et al., } 2014\end{array}$ & 4 & $\begin{array}{l}20 \mathrm{~min} \text { (totally } \\
20 \mathrm{~min}, 20 \mathrm{~min} \\
\text { for left foot) }\end{array}$ & 1 & 4 (1.33 hours) & $\begin{array}{l}\text { Not } \\
\text { reported }\end{array}$ & Not reported & $40 / 40=100 \%$ \\
\hline $\begin{array}{l}\text { Khaledifar et al., } \\
2017\end{array}$ & 1 & $\begin{array}{l}30 \mathrm{~min} \text { (totally } \\
30 \mathrm{~min}, 15 \mathrm{~min} \\
\text { for each foot) }\end{array}$ & 1 & 1 (0.5 hours) & $\begin{array}{l}\text { Not } \\
\text { reported }\end{array}$ & Not reported & $25 / 25=100 \%$ \\
\hline Saatsaz et al., 2016 & 1 & - & 1 & $1(-)$ & $\begin{array}{l}\text { Not } \\
\text { reported }\end{array}$ & $\begin{array}{l}90 \text { min after foot } \\
\text { massage }\end{array}$ & $52 / 52=100 \%$ \\
\hline Pasyar et al., 2018 & 1 & $\begin{array}{l}10 \text { (total } 10 \mathrm{~min} \text {, } \\
5 \mathrm{~min} \text { for each } \\
\text { foot) }\end{array}$ & 1 & 1 (0.16 hours) & $\begin{array}{l}\text { Not } \\
\text { reported }\end{array}$ & $\begin{array}{l}2 \text { hours after } \\
\text { foot massage }\end{array}$ & $33 / 33=100 \%$ \\
\hline Koras et al., 2019 & 1 & $\begin{array}{l}40 \text { (total } 40 \mathrm{~min} \\
20 \mathrm{~min} \text { for each } \\
\text { foot) }\end{array}$ & 1 & 1 (0.66 hour) & $\begin{array}{l}\text { Not } \\
\text { reported }\end{array}$ & $\begin{array}{l}90 \text { min after foot } \\
\text { massage }\end{array}$ & $85 / 85=100 \%$ \\
\hline Eguchi et al., 2016 & 3 & $45 \mathrm{~min}$ & 4 & 12 (9 hours) & $\begin{array}{l}\text { No side } \\
\text { effect }\end{array}$ & Not reported & $27 / 27=100 \%$ \\
\hline
\end{tabular}


TABLE 2: Continued.

\begin{tabular}{|c|c|c|c|c|c|c|c|}
\hline Authors, year & $\begin{array}{c}\text { Frequency } \\
\text { (sessions/ } \\
\text { week) }\end{array}$ & $\begin{array}{l}\text { Session length } \\
\text { (mins/session) }\end{array}$ & $\begin{array}{c}\text { Duration } \\
\text { (weeks/ } \\
\text { study) } \\
\end{array}$ & $\begin{array}{c}\text { Number of } \\
\text { sessions/study } \\
\text { total length/study }\end{array}$ & $\begin{array}{c}\text { Safety } \\
\text { (adverse } \\
\text { events) }\end{array}$ & $\begin{array}{l}\text { Lasting effects } \\
\text { and duration }\end{array}$ & $\begin{array}{l}\text { Adherence } \\
\text { rate (\%) }\end{array}$ \\
\hline Ozturk et al., 2018 & 3 & $\begin{array}{l}20 \text { (total } 20 \mathrm{~min}, \\
10 \mathrm{~min} \text { for each } \\
\text { foot) }\end{array}$ & 1 & 3 (1 hour) & $\begin{array}{l}\text { Not } \\
\text { reported }\end{array}$ & Not reported & $32 / 32=100 \%$ \\
\hline $\begin{array}{l}\text { Ramezanibadr } \\
\text { et al., } 2018\end{array}$ & 1 & $20 \mathrm{~min}$ & 1 & 1 (0.33 hour) & $\begin{array}{c}\text { Not } \\
\text { reported }\end{array}$ & $\begin{array}{l}1 \text { hour after foot } \\
\text { reflexology }\end{array}$ & $50 / 50=100 \%$ \\
\hline $\begin{array}{l}\text { Shahsavari et al., } \\
2017\end{array}$ & 1 & $30 \mathrm{~min}$ & 1 & 1 (0.5 hour) & $\begin{array}{l}\text { Not } \\
\text { reported }\end{array}$ & Not reported & $40 / 40=100 \%$ \\
\hline $\begin{array}{l}\text { Abbaszadeh et al., } \\
2018\end{array}$ & 4 & $\begin{array}{l}30 \text { (total } 30 \mathrm{~min} \text {, } \\
15 \mathrm{~min} \text { for each } \\
\text { foot) }\end{array}$ & 1 & 4 (2 hours) & $\begin{array}{l}\text { Not } \\
\text { reported }\end{array}$ & Not reported & $40 / 40=100 \%$ \\
\hline Levy et al., 2020 & 1 & 30 (total $30 \mathrm{~min})$ & 1 & 1 (0.5 hour) & $\begin{array}{l}\text { No side } \\
\text { effect }\end{array}$ & Not reported & $99 / 99=100 \%$ \\
\hline
\end{tabular}

TABLE 3: Risk of the methodological bias score of included studies.

\begin{tabular}{|c|c|c|c|c|c|c|c|}
\hline Authors, year & $\begin{array}{c}\text { Random } \\
\text { sequence } \\
\text { generation } \\
\text { (selection bias) }\end{array}$ & $\begin{array}{c}\text { Allocation } \\
\text { concealment } \\
\text { (selection bias) }\end{array}$ & $\begin{array}{c}\text { Binding of } \\
\text { participants and } \\
\text { personnel } \\
\text { (performance bias) }\end{array}$ & $\begin{array}{l}\text { Blinding of } \\
\text { outcome } \\
\text { assessment } \\
\text { (detecting bias) }\end{array}$ & $\begin{array}{c}\text { Incomplete } \\
\text { outcome data } \\
\text { (attrition bias) }\end{array}$ & $\begin{array}{l}\text { Selective } \\
\text { reporting bias } \\
\text { (reporting } \\
\text { bias) }\end{array}$ & $\begin{array}{c}\text { Other } \\
\text { bias }\end{array}$ \\
\hline Valizadeh, 2015 & $\mathrm{U}$ & $\mathrm{U}$ & $\mathrm{H}$ & $\mathrm{U}$ & $\mathrm{L}$ & $\mathrm{L}$ & $\mathrm{H}$ \\
\hline Li, 2011 & $\mathrm{~L}$ & $\mathrm{U}$ & $\mathrm{H}$ & $\mathrm{H}$ & $\mathrm{L}$ & $\mathrm{L}$ & $\mathrm{U}$ \\
\hline Bakir, 2018 & $\mathrm{U}$ & $\mathrm{U}$ & $\mathrm{U}$ & $\mathrm{U}$ & $\mathrm{L}$ & $\mathrm{L}$ & $\mathrm{H}$ \\
\hline Unal, 2016 & $\mathrm{U}$ & $\mathrm{U}$ & $\mathrm{U}$ & $\mathrm{U}$ & $\mathrm{L}$ & $\mathrm{L}$ & $\mathrm{H}$ \\
\hline Zengin, 2018 & $\mathrm{~L}$ & $\mathrm{U}$ & $\mathrm{U}$ & $\mathrm{U}$ & $\mathrm{L}$ & $\mathrm{L}$ & $\mathrm{U}$ \\
\hline Rambod, 2019 & $\mathrm{~L}$ & $\mathrm{~L}$ & $\mathrm{H}$ & $\mathrm{L}$ & $\mathrm{L}$ & $\mathrm{L}$ & $\mathrm{U}$ \\
\hline $\begin{array}{l}\text { Malekshahi, } \\
2018\end{array}$ & $\mathrm{U}$ & $\mathrm{U}$ & $\mathrm{U}$ & $\mathrm{U}$ & $\mathrm{U}$ & $\mathrm{L}$ & $\mathrm{H}$ \\
\hline Oshvandi, 2014 & $\mathrm{~L}$ & $\mathrm{~L}$ & $\mathrm{H}$ & $\mathrm{U}$ & $\mathrm{L}$ & $\mathrm{L}$ & $\mathrm{U}$ \\
\hline $\begin{array}{l}\text { Samarehfekri, } \\
2020\end{array}$ & $\mathrm{~L}$ & $\mathrm{~L}$ & $\mathrm{U}$ & $\mathrm{U}$ & $\mathrm{L}$ & $\mathrm{L}$ & $\mathrm{U}$ \\
\hline Toygar, 2020 & $\mathrm{~L}$ & $\mathrm{U}$ & $\mathrm{L}$ & $\mathrm{L}$ & $\mathrm{L}$ & $\mathrm{L}$ & $\mathrm{U}$ \\
\hline Bahrami, 2019 & $\mathrm{~L}$ & $\mathrm{~L}$ & $\mathrm{H}$ & $\mathrm{U}$ & $\mathrm{L}$ & $\mathrm{L}$ & $\mathrm{U}$ \\
\hline Noh et al., 2019 & $\mathrm{~L}$ & $\mathrm{U}$ & $\mathrm{H}$ & $\mathrm{U}$ & $\mathrm{L}$ & $\mathrm{L}$ & $\mathrm{U}$ \\
\hline $\begin{array}{l}\text { Mahdavipour } \\
\text { et al., } 2019\end{array}$ & $\mathrm{U}$ & $\mathrm{U}$ & $\mathrm{H}$ & $\mathrm{U}$ & $\mathrm{L}$ & $\mathrm{L}$ & $\mathrm{U}$ \\
\hline $\begin{array}{l}\text { Soheili et al., } \\
2017\end{array}$ & $\mathrm{~L}$ & $\mathrm{U}$ & $\mathrm{H}$ & $\mathrm{U}$ & $\mathrm{L}$ & $\mathrm{L}$ & $\mathrm{U}$ \\
\hline $\begin{array}{l}\text { Vardanjani et al., } \\
2013\end{array}$ & $\mathrm{~L}$ & $\mathrm{U}$ & $\mathrm{H}$ & $\mathrm{U}$ & $\mathrm{L}$ & $\mathrm{L}$ & $\mathrm{H}$ \\
\hline $\begin{array}{l}\text { Bagheri-nesami } \\
\text { et al., } 2014\end{array}$ & $\mathrm{~L}$ & $\mathrm{U}$ & $\mathrm{U}$ & $\mathrm{U}$ & $\mathrm{L}$ & $\mathrm{L}$ & $\mathrm{U}$ \\
\hline $\begin{array}{l}\text { Khaledifar et al., } \\
2017\end{array}$ & $\mathrm{U}$ & $\mathrm{U}$ & $\mathrm{U}$ & $\mathrm{U}$ & $\mathrm{L}$ & $\mathrm{L}$ & $\mathrm{H}$ \\
\hline $\begin{array}{l}\text { Saatsaz et al., } \\
2016\end{array}$ & $\mathrm{~L}$ & $\mathrm{U}$ & $\mathrm{H}$ & $\mathrm{U}$ & $\mathrm{L}$ & $\mathrm{L}$ & $\mathrm{H}$ \\
\hline $\begin{array}{l}\text { Pasyar et al., } \\
2018\end{array}$ & $\mathrm{~L}$ & $\mathrm{~L}$ & $\mathrm{H}$ & $\mathrm{U}$ & $\mathrm{L}$ & $\mathrm{L}$ & $\mathrm{H}$ \\
\hline Koras et al., 2019 & $\mathrm{U}$ & $\mathrm{U}$ & $\mathrm{H}$ & $\mathrm{U}$ & $\mathrm{L}$ & $\mathrm{L}$ & $\mathrm{H}$ \\
\hline $\begin{array}{l}\text { Eguchi et al., } \\
2016\end{array}$ & $\mathrm{U}$ & $\mathrm{U}$ & $\mathrm{U}$ & $\mathrm{U}$ & $\mathrm{L}$ & $\mathrm{L}$ & $\mathrm{U}$ \\
\hline $\begin{array}{l}\text { Ozturk et al., } \\
2018\end{array}$ & $\mathrm{~L}$ & $\mathrm{U}$ & $\mathrm{H}$ & $\mathrm{U}$ & $\mathrm{L}$ & $\mathrm{L}$ & $\mathrm{U}$ \\
\hline $\begin{array}{l}\text { Ramezanibadr } \\
\text { et al., } 2018\end{array}$ & $\mathrm{~L}$ & $\mathrm{U}$ & $\mathrm{H}$ & $\mathrm{U}$ & $\mathrm{L}$ & $\mathrm{L}$ & $\mathrm{U}$ \\
\hline $\begin{array}{l}\text { Shahsavari et al., } \\
2017\end{array}$ & $\mathrm{~L}$ & $\mathrm{U}$ & $\mathrm{H}$ & $\mathrm{U}$ & $\mathrm{L}$ & $\mathrm{L}$ & $\mathrm{U}$ \\
\hline $\begin{array}{l}\text { Abbaszadeh } \\
\text { et al., } 2018\end{array}$ & $\mathrm{~L}$ & $\mathrm{~L}$ & $\mathrm{U}$ & $\mathrm{L}$ & $\mathrm{L}$ & $\mathrm{L}$ & $\mathrm{U}$ \\
\hline Levy et al., 2020 & $\mathrm{~L}$ & $\mathrm{U}$ & $\mathrm{H}$ & $\mathrm{L}$ & $\mathrm{L}$ & $\mathrm{L}$ & $\mathrm{U}$ \\
\hline
\end{tabular}




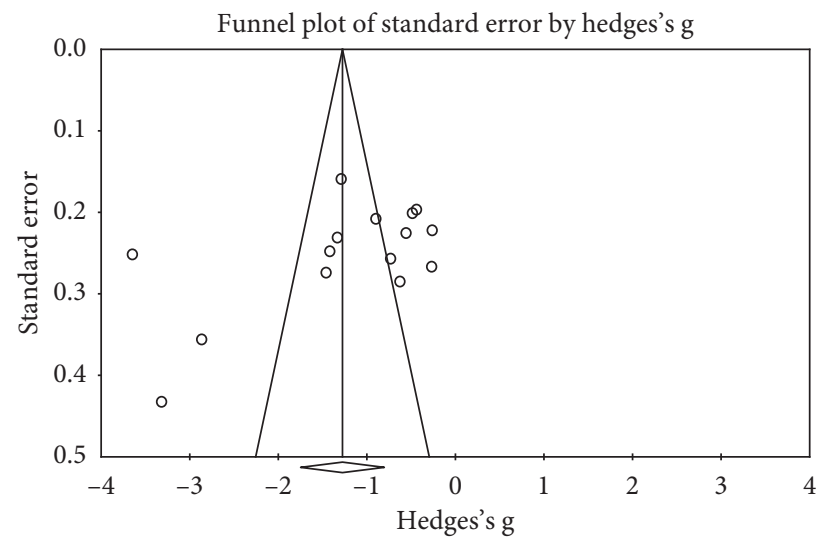

FIgURE 2: Visual inspection of the funnel plot for effect for improving anxiety symptom.

TABle 4: Overall effect size of foot reflexology intervention for an adult.

\begin{tabular}{lccccccccrr}
\hline & & Effect size & \multicolumn{2}{c}{$95 \%$ CI } & \multicolumn{2}{c}{ Null hypothesis } & \multicolumn{3}{c}{ Heterogeneity } \\
& & & & \multicolumn{2}{c}{ Two-tailed test } & & \\
& Sample size (studies) & Hedge's $g$ & Lower & Upper & $Z$ value & $P$ Value & $Q$ value & $P$ value & $I^{2}$ \\
\hline Depression & 4 & -0.921 & -1.246 & -0.595 & -5.542 & $<0.001$ & 5.42 & 0.143 & 44.74 \\
Anxiety & 16 & -1.237 & -1.682 & -0.791 & -5.435 & $<0.001$ & 217.41 & $<0.001$ & 93.10 \\
Sleep quality & 10 & -1.665 & -2.361 & -0.970 & -4.692 & $<0.001$ & 144.87 & $<0.001$ & 93.78 \\
\hline
\end{tabular}

$P$ values $>0.001$ were rounded to two digits. CI, confidence interval.

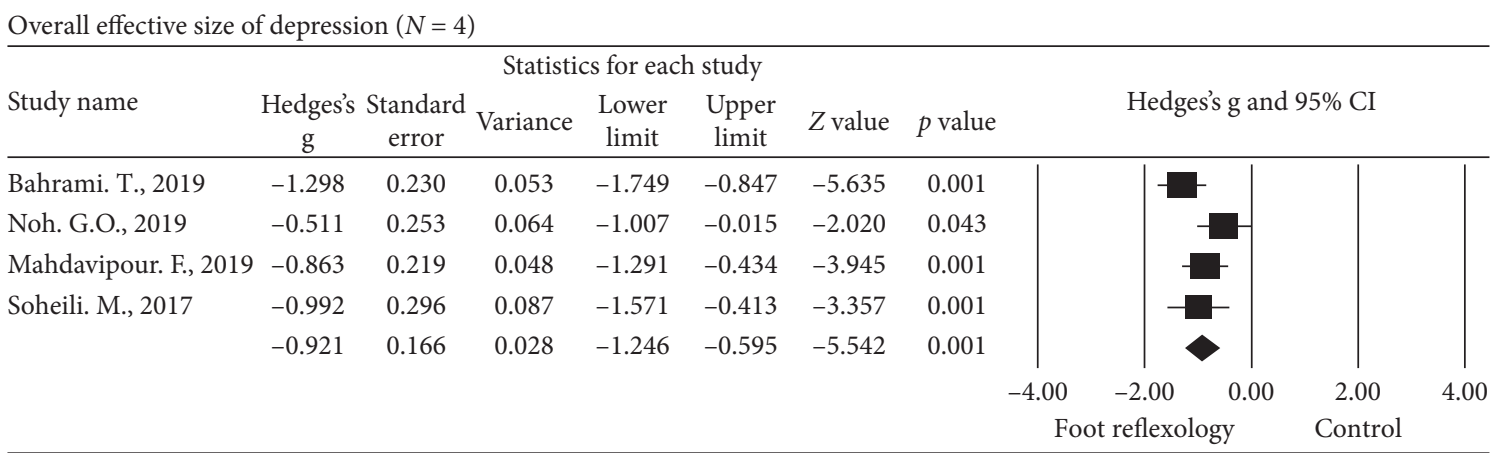

FIgURE 3: Overall effect size of the improvement of depression in adults following foot reflexology intervention ( $n=4$ studies).

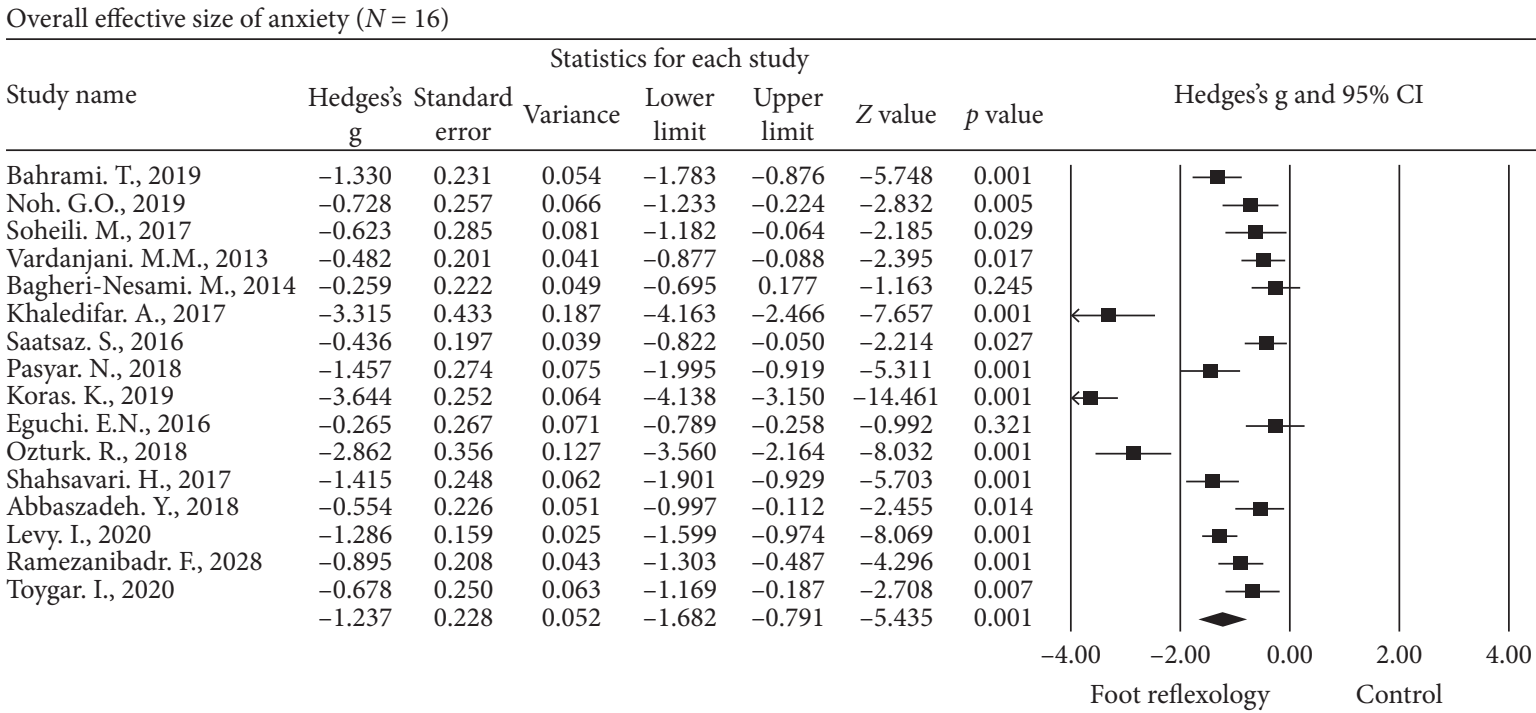

FIGURE 4: Overall effect size of the improvement of anxiety in adults following foot reflexology intervention ( $n=16$ studies). 


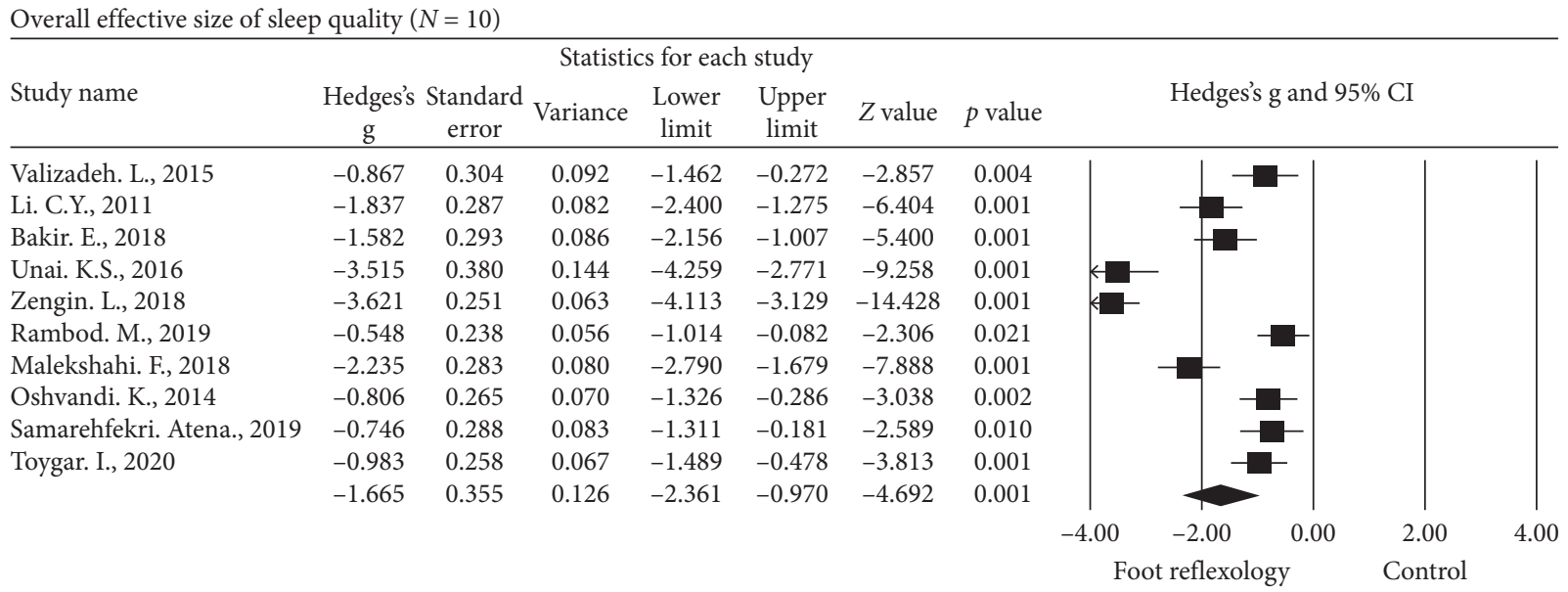

FIGURE 5: Overall effect size of the improvement of sleep quality in adults following foot reflexology intervention ( $n=10$ studies).

reporting of adverse events and personal reasoning for dropouts from participants.

4.2. Comparison with Prior Reviews. No systematic review explicitly focusing on foot reflexology for improving psychological symptoms including depression, anxiety, and sleep quality was accessible. Ours is the first systematic review and meta-analysis with 26 RCTs to focus on the effects of foot reflexology on depression, anxiety, and sleep quality. We identified there is no direct correlation or evidence on previous meta-analysis reports on self-administered foot reflexology with subjective and objective outcomes for healthy persons [65], benefits of foot reflexology for insomnia [66], or effects of foot reflexology on fatigue, sleep, and pain [67]. The studies of these analyses are nonrandomized trials before-and-after studies, and the sample size of these studies was too small. Future research should ensure detailed and precise methodology and adequate sample size to better evaluate the impact of foot reflexology intervention. Results of previous reviews published on 2019 reveal effectiveness of reflexology intervention on premenstrual syndrome [68] and anxiety of patients undergoing cardiovascular interventional procedures [69]. These two recent reviews illustrated that all reflexology intervention practices including hand reflexology and foot reflexology benefited participants in specific groups. Our meta-analysis with 26 RCTs emphasized foot reflexology intervention on depression, anxiety, and sleep quality and conducted further exploration on the determinants of the heterogeneity with subgroup analysis for both categorical and continuous moderators to find significant factors for perceived heterogeneity.

4.3. External and Internal Validity. Major threats to external validity included specific variables of sampled participants and multiple foot reflexology intervention types. The majority of RCTs included participants from Asia. The lack of studies from America, Europe, and Africa was apparent. It might not be applicable to other areas. Heterogeneity is high due to wide variability in participant groups, foot reflexology technique, selection of reflexology zones, foot reflexology intervention duration, and frequency.

Internal validity is limited due to the methodological quality of the included studies. All of the included studies used self-reported questionnaires for depression, anxiety, and sleep quality; thus, recall bias could not be excluded. It remains to be determined whether differences in these parameters could affect results. All of our studies asserted that they had applied randomization methods; however, not all of the studies elaborate on the design protocol and methods of randomization, and some of the included studies seem to not have been truly randomized. It also proves difficult to properly blinding. Only one of the reviewed RCTs successfully implemented blinding in the participants [62]. Erroneous random sequence generation and allocation concealment have been empirically revealed to be a significant source of bias in RCTs [70]. Our included studies only had a low risk or an unclear risk of selection bias with no high risk selection bias. All the effects were robust against potential risk of selection bias, and the internal validity of the review, while limited, is still acceptable.

4.4. Strengths and Weaknesses. This is the first and latest comprehensive systematic review and meta-analysis available on foot reflexology for depression, anxiety, and sleep quality with a large number of randomized controlled trials. None of studies provided any adverse effects of foot reflexology, thereby indicating the importance of using foot reflexology as an effective and less complicated intervention practice. There were seven primary limitations of this review [71]. First, despite great efforts to locate all relevant RCTs of foot reflexology intervention for psychological symptoms, there may be a degree of uncertainty due to a limitation in interlanguage communication, limited resources, and bias in publication. Due to language constraints, we did not include Arab States, Japanese, and Korean database. Second, only one of the studies provided the methods of blinding. Participant blinding is sometimes impossible to fully control; for example, trials in sport, surgical intervention, 
TABLE 5: Mean effect sizes and moderator analyses of foot reflexology intervention.

\begin{tabular}{|c|c|c|c|c|}
\hline & Parameter & Results & Effect sizes (Hedges' $g$ ) & $95 \% \mathrm{CI}$ \\
\hline \multirow{20}{*}{ Anxiety } & Categorical moderators & & & \\
\hline & Outcome measurement tool & & & \\
\hline & STAI & 9 & -1.534 & $-2.332,-0.736$ \\
\hline & Others & 7 & -0.894 & $-1.241,-0.547$ \\
\hline & Reflexology before/after & & & \\
\hline & Surgical intervention & & & \\
\hline & Before & 5 & -1.409 & $-2.083,-0.735$ \\
\hline & After & 5 & -1.745 & $-3.066,-0.427$ \\
\hline & Intervention type & & & \\
\hline & 1 time intervention & 9 & -1.553 & $-2.190,-0.915$ \\
\hline & $>1$ time intervention & 6 & -0.849 & $-1.471,-0.226$ \\
\hline & Surgical intervention type & & & \\
\hline & Cardiovascular intervention & 5 & -1.060 & $-1.652,-0.467$ \\
\hline & Other surgery & 5 & -2.340 & $-3.485,-1.195$ \\
\hline & Random sequence generation & & & \\
\hline & High/unclear risk & 3 & -2.401 & $-4.737,-0.064$ \\
\hline & Low risk & 13 & -0.970 & $-1.275,-0.666$ \\
\hline & Allocation concealment & & & \\
\hline & High/unclear risk & 12 & -1.271 & $-1.812,-0.730$ \\
\hline & Low risk & 3 & -1.102 & $-1.668,-0.536$ \\
\hline \multirow{12}{*}{ Sleep quality } & Outcome measurement tool & & & \\
\hline & PSQI & 7 & -2.021 & $-2.931,-1.112$ \\
\hline & Others & 3 & -0.853 & $-1.158,-0.548$ \\
\hline & Participant & & - & \\
\hline & Hemodialysis group & 2 & 2.850 & $-4.104,-1.596$ \\
\hline & Nonhemodialysis group & 8 & -1.375 & $-2.119,-0.632$ \\
\hline & Random sequence generation & & & \\
\hline & High/unclear risk & 4 & -2.032 & $-3.033,-1.031$ \\
\hline & Low risk & 6 & -1.424 & $-2.395,-0.454$ \\
\hline & Allocation concealment & & & \\
\hline & High/unclear risk & 7 & -2.085 & $-2.913,-1.257$ \\
\hline & Low risk & 3 & -0.686 & $-0.981,-0.390$ \\
\hline \multirow{4}{*}{ Anxiety } & Parameter & Results & Slope & $95 \% \mathrm{CI}$ \\
\hline & Continuous moderators & & & \\
\hline & Mean age & 11 & -0.155 & $-1.790,1.480$ \\
\hline & Total length in one time & 8 & -0.126 & $-2.217,1.966$ \\
\hline \multirow{3}{*}{ Sleep quality } & Mean age & 8 & 0.035 & $-0.049,0.056$ \\
\hline & Total length of time & 10 & -0.346 & $-0.568,-0.124$ \\
\hline & Duration & 10 & -0.256 & $-0.466,-0.046$ \\
\hline
\end{tabular}

nonpharmacological therapy, were all not valued as appropriate, lacking pragmatic and systemic aim. Previous studies provided empirical evidence of pronounced bias due to deficiency in patient bias control in related randomized clinical trials with patient-reported outcomes [72]. Third, the critical flaw of this study was the relative lack of high-quality RCTs. The small number of participating studies meant that the statistical power to detect differences was suboptimal. Future large-scale trials may be recommended to demonstrate this effect. Fourth, masseuses often chat with their clients which has a psychological effect which may influence this research. Social interaction has been known to reduce stress and anxiety. If some practitioners speak to their clients while others do not, this would impact results greatly. Control over social interaction is needed for further research. The fifth limitation is the severity of the complaints concerning psychological symptoms and health status of the participants. This was not considered appropriate and was not individually listed in each study. Differences in selfreported questionnaires were found between intervention and control groups in some studies. This may have led to heterogeneity. The sixth limitation was that the intensity (size of strength), frequency (sessions of per week), and duration (time of each session) of foot reflexology interventions were all heterogeneous. Most of the studies were short-term applications without long-term follow-up effects. Lastly, a lack of priority in safety evaluation may have caused each study to produce minimal occurrences in serious adverse events or nonserious events. It only can be assumed that foot reflexology intervention is a low-risk treatment option.

4.5. Implications for Further Research. If possible, we should expand research parameters to include western countries such as Canada or the United States. Different countries 
may include foot reflexology under their national health insurance or private healthcare plans. If a client has free access to this treatment, they naturally are more inclined to continue (adherence rate would increase). However, if foot reflexology is not covered under a client's healthcare provided, they would be less likely to continue (adherence rate would decrease). This systematic review and metaanalysis were limited by the low methodological quality of included studies. Further RCTs should enforce thorough methodology and reports, which would mean appropriate sample size, adequate randomization, allocation concealment, intention-to-treat analysis, and bias control of the least one outcome assessors [73]. In order to achieve successful bias control of participants and minimize any physiological effects, a physical force less than the minimal force is required in foot reflexology at nonreflexology areas and may be regarded as a sham control. According to the funnel plots, there could be a publication bias in which authors lose confidence in their published trials if their results produced negative conclusions. The quality of the results of meta-analysis was determined by the quality of the RCT and by sufficient clinical evidence. Thus, if we want to draw a reasonable conclusion for a meta-analysis, we need larger sample sizes and more rigorously randomized controlled trials. Researchers for study interventions may need to apply a standard protocol to specific demographic group. Objective psychological symptoms measuring tools, such as actigraphy or heart rate variability analysis, should be incorporated to more accurately evaluate the effect of foot reflexology. There is a lack of evidence in follow-up effects of foot reflexology in psychological symptoms. So, long-term follow-ups should be necessary in future RCTs. Ample reporting of safety issues with foot reflexology intervention should be utilized in future randomized controlled trials. Limited evidence impaired our research because no studies reported safetyrelated adverse effects. Most of the included studies failed to report on this aspect.

\section{Conclusions}

Results of this systematic review and meta-analysis demonstrated that foot reflexology intervention has benefits compared to nonactive control practices in terms of ameliorate the burden of depression, anxiety, and sleep disturbance. Furthermore, metaregression reveals that an increase in total foot reflexology time would decrease anxiety and improve sleep quality. Despite certain flaws in methodology in our included studies, foot reflexology may be recommended as a complementary intervention to improve our depression, anxiety, and sleep quality. However, advanced strength of evidence with future understanding of the mechanisms of foot reflexology and long-term follow-up should be a priority for future preparation and implementation for sensitive groups, such as delivering women or cancer patients, who may be unable to use other means of care and benefit from such care.

\section{Data Availability}

The data used to support the findings of this study are included within the article.

\section{Conflicts of Interest}

The authors declare that they do not have any conflicts of interest with the conducted research.

\section{Authors' Contributions}

Wei-Li Wang and Ying-Ren Chen conceived and designed the experiments. Wei-Li Wang, Ying-Ren Chen, Hao-Yuan Hung, Yuan-Yu Chan, Kuang-Huei Chen, and Szu-Nian Yang analyzed the data. Hao-Yuan Hung, Yuan-Yu Chan, Chi-Ming Chu. Wrote the paper: Wei-Li Wang, Hao-Yuan Hung, and Yuan-Yu Chan contributed reagents/materials/analysis tools.

\section{Acknowledgments}

The authors acknowledge the grant support from the Taoyuan Armed Forces General Hospital, Taiwan (TYAFGH-E-109056). This funding agency did not influence the study design, data collection and analysis, decision to publish, or preparation of the manuscript.

\section{References}

[1] Y. M. Lee, "Effect of self-foot reflexology massage on depression, stress responses and immune functions of middle aged women," Journal of Korean Academy of Nursing, vol. 36, no. 1, pp. 179-188, 2016.

[2] T. Evyapan, Reflexology for Hands and Feet, Arkadas Publishing, Ankara, Turkey, 2012.

[3] K. Cevik, "Complementary and alternative therapies in nursing: reflexology," International Journal of Nursing, vol. 29, pp. 71-82, 2015.

[4] N. H. Embong, Y. C. Soh, L. C. Ming, and T. W. Wong, "Perspectives on reflexology: a qualitative approach," Journal of Traditional and Complementary Medicine, vol. 7, no. 3, pp. 327-331, 2017.

[5] C. M. Hughes, S. Krirsnakriengkrai, S. Kumar, and S. M. McDonough, "The effect of reflexology on the autonomic nervous system in healthy adults: a feasibility study," Alternative Therapies in Health and Medicine, vol. 17, no. 3, pp. 32-37, 2011.

[6] D. Tiran and H. Chummun, "The physiological basis of reflexology and its use as a potential diagnostic tool," Complementary Therapies in Clinical Practice, vol. 11, no. 1, pp. 58-64, 2005.

[7] F. Mahdavipour, Z. Rahemi, Z. Sadat, and N. M. Ajorpaz, "The effects of foot reflexology on depression during menopause: a randomized controlled clinical trial," Complementary Therapies in Medicine, vol. 47, Article ID 102195, 2019.

[8] L. Zengin and R. Aylaz, "The effects of sleep hygiene education and reflexology on sleep quality and fatigue in patients receiving chemotherapy," European Journal of Cancer Care, vol. 28, no. 3, Article ID e13020, 2019.

[9] A. Ozdelikara and M. Tan, "The effect of reflexology on the quality of life with breast cancer patients," Complementary Therapies in Clinical Practice, vol. 29, pp. 122-129, 2017. 
[10] A. Holbrook, R. Crowther, A. Lotter, and Y. Endeshaw, "The role of benzodiazepines in the treatment of insomnia: meta-analysis of benzodiazepine use in the treatment of insomnia," Journal of the American Geriatrics Society, vol. 49, no. 6, pp. 824-826, 2011.

[11] I. Dougans, Complete Illustrated Guide to Reflexology, Element, London, UK, 2nd edition, 1996.

[12] J. E. McCullough, S. D. Liddle, M. Sinclair, C. Close, and C. M. Hughes, "The physiological and biochemical outcomes associated with a reflexology treatment: a systematic review," Evidence-Based Complementary and Alternative Medicine, vol. 2013, Article ID 502123, 16 pages, 2013.

[13] D. Moher, A. Liberati, J. Tetzlaff, D. G. Altman, and P. Group, "Preferred reporting items for systematic reviews and metaanalyses: the prisma statement," PLoS Medicine, vol. 6, no. 7, Article ID e1000097, 2009.

[14] J. Higgins and S. Green, Cochrane Handbook for Systematic Reviews of Interventions, Wiley, Hoboken, NJ, USA, 2008.

[15] Food and Drug Administration (FDA), What is a Serious Adverse Event?, Food and Drug Administration (FDA), White Oak, MD, USA, 2016.

[16] J. P. T. Higgins and G. A. Douglas, "Assessing risk of bias in included studies," Assessing risk of bias in included studies," in Cochrane Handbook for Systematic Reviews of Interventions, P. T. H. Julian and S. Green, Eds., Wiley-Blackwell, Chichester, UK, pp. 187-241, 2018.

[17] M. Borenstein, L. Hedges, and H. Rothstein, Introduction to Meta-Analysis, Wiley, Chichester, England, 2009.

[18] J. Cohen, Statistical Power Analysis for the Behavioral Science, Lawrence Erlbaum Associates, Hillsdale, NJ, USA, 2nd edition, 1969.

[19] M. Egger, G. Davey Smith, M. Schneider, and C. Minder, "Bias in meta-analysis detected by a simple, graphical test," BMJ, vol. 315, no. 7109, pp. 629-634, 1997.

[20] A. Abbasi Fakhravari, F. Bastani, and H. Haghani, "The effect of foot reflexology massage on the sleep quality of elderly women with restless leg syndrome," Journal of Client-Centered Nursing Care, vol. 4, pp. 96-103, 2018.

[21] M. Asltoghiri and Z. Ghodsi, "The effects of reflexology on sleep disorder in menopausal women," Procedia-Social and Behavioral Sciences, vol. 31, pp. 242-246, 2012.

[22] N. L. Stephenson, S. P. Weinrich, and A. S. Tavakoli, "The effects of foot reflexology on anxiety and pain in patients with breast and lung cancer," Oncology Nursing Forum, vol. 27, no. 1, pp. 67-72, 2000.

[23] A. Ozdelikara and S. Agcadiken Alkan, "The effects of reflexology on fatigue and anxiety in patients with multiple sclerosis," Alternative Therapies in Health and Medicine, vol. 24, no. 4, pp. 8-13, 2018.

[24] A. Kheyri, F. Bastani, and H. Haghani, "Effect of reflexology on sleep quality of elderly women undergoing abdominal surgery," Journal of Client-Centered Nursing Care, vol. 2, pp. 11-18, 2016.

[25] A. Rahmani, M. Naseri, M. M. Salaree, and B. Nehrir, "Comparing the effect of foot reflexology massage, foot bath and their combination on quality of sleep in patients with acute coronary syndrome," Journal of Caring Sciences, vol. 5, no. 4, pp. 299-306, 2016.

[26] R. Tarrasch, N. N. Carmel Neiderman, S. Ben Ami et al., "The effect of reflexology on the pain-insomnia-fatigue disturbance cluster of breast cancer patients during adjuvant radiation therapy," The Journal of Alternative and Complementary Medicine, vol. 24, no. 1, pp. 62-68, 2018.
[27] Y. Aydin, E. Aslan, and O. Yalcin, "Effect of reflexology to depressive symptoms in women with overactive bladder," Holistic Nursing Practice, vol. 30, no. 5, pp. 294-300, 2016.

[28] S. Moghimi Hanjani, Z. Mehdizadeh Tourzani, and M. Shoghi, "The effect of foot reflexology on anxiety, pain, and outcomes of the labor in primigravida women," Acta Medica Iranica, vol. 53, no. 8, pp. 507-511, 2015.

[29] Z. Yilar Erkek and S. Aktas, "The effect of foot reflexology on the anxiety levels of women in labor," The Journal of Alternative and Complementary Medicine, vol. 24, no. 4, pp. 352-360, 2018.

[30] G. Mahmoudirad, M. G. Moslo, and H. Bahrami, "Effect of foot reflexology on anxiety of patients undergoing coronary angiography," Iranian Journal of Critical Care Nursing, vol. 4, pp. 235-242, 2014.

[31] J. E. M. McCullough, S. D. Liddle, C. Close, M. Sinclair, and C. M. Hughes, "Reflexology: a randomised controlled trial investigating the effects on beta-endorphin, cortisol and pregnancy related stress," Complementary Therapies in Clinical Practice, vol. 31, pp. 76-84, 2018.

[32] G. Wyatt, A. Sikorskii, I. Tesnjak et al., "A randomized clinical trial of caregiver-delivered reflexology for symptom management during breast cancer treatment," Journal of Pain and Symptom Management, vol. 54, no. 5, pp. 670-679, 2017.

[33] J. Williamson, A. White, A. Hart, and E. Ernst, "Randomised controlled trial of reflexology for menopausal symptoms," BJOG, vol. 109, no. 9, pp. 1050-1055, 2002.

[34] N. Cheraghbeigi, M. Modarresi, M. Rezaei, and A. Khatony, "Comparing the effects of massage and aromatherapy massage with lavender oil on sleep quality of cardiac patients: a randomized controlled trial," Complementary Therapies in Clinical Practice, vol. 35, pp. 253-258, 2019.

[35] S. Azima, H. R. Bakhshayesh, M. Kaviani, K. Abbasnia, and M. Sayadi, "Comparison of the effect of massage therapy and isometric exercises on primary dysmenorrhea: a randomized controlled clinical trial," Journal of Pediatric and Adolescent Gynecology, vol. 28, no. 6, pp. 486-491, 2015.

[36] K. K. Sozen and N. Karabulut, "Efficacy of hand and foot massage in anxiety and pain management following laparoscopic cholecystectomy: a controlled randomized study," Surgical Laparoscopy, Endoscopy \& Percutaneous Techniques, vol. 30, no. 2, 2020.

[37] H. S. Alimohammad, Z. Ghasemi, S. Shahriar, S. Morteza, and K. Arsalan, "Effect of hand and foot surface stroke massage on anxiety and vital signs in patients with acute coronary syndrome: a randomized clinical trial," Complement Ther Clin Pract, vol. 31, pp. 126-131, 2018.

[38] J. Hattan, L. King, and P. Griffiths, "The impact of foot massage and guided relaxation following cardiac surgery: a randomized controlled trial," Journal of Advanced Nursing, vol. 37, no. 2, pp. 199-207, 2002.

[39] S. Dehghanmehr, G. H. Sargazi, A. Biabani, S. Nooraein, and J. Allahyari, "Comparing the effect of acupressure and foot reflexology on anxiety and depression in hemodialysis patients: a clinical trial," Medical-Surgical Nursing Journal, vol. 8, 2020.

[40] F. Davodabady, V. Naseri-Salahshour, M. Sajadi, A. Mohtarami, and F. Rafiei, "Randomized controlled trial of the foot reflexology on pain and anxiety severity during dressing change in burn patients," Burns, 2020.

[41] L. Valizadeh, A. Seyyedrasooli, V. Zamanazadeh, and K. Nasiri, "Comparing the effects of reflexology and footbath on sleep quality in the elderly: a controlled clinical trial," Iranian Red Crescent Medical Journal, vol. 17, no. 11, Article ID e20111, 2015. 
[42] E. Bakir, S. S. Baglama, and S. Gursoy, "The effects of reflexology on pain and sleep deprivation in patients with rheumatoid arthritis: a randomized controlled trial," Complementary Therapies in Clinical Practice, vol. 31, pp. 315-319, 2018.

[43] K. S. Unal and R. Balci Akpinar, "The effect of foot reflexology and back massage on hemodialysis patients' fatigue and sleep quality," Complementary Therapies in Clinical Practice, vol. 24, pp. 139-144, 2016.

[44] F. Malekshahi, F. Aryamanesh, and S. Fallahi, "The effects of massage therapy on sleep quality of patients with end-stage renal disease undergoing hemodialysis," Sleep and Hypnosis-International Journal, vol. 2, pp. 91-95, 2017.

[45] A. Khaledifar, M. Nasiri, B. Khaledifar, A. Khaledifar, and A. Mokhtari, "The effect of reflexotherapy and massage therapy on vital signs and stress before coronary angiography: an open-label clinical trial," ARYA Atherosclerosis, vol. 13, no. 2, pp. 50-55, 2017.

[46] K. Koras and N. Karabulut, "The effect of foot massage on postoperative pain and anxiety levels in laparoscopic cholecystectomy surgery: a randomized controlled experimental study," Journal of PeriAnesthesia Nursing, vol. 34, no. 3, pp. 551-558, 2019.

[47] E. Eguchi, N. Funakubo, K. Tomooka, T. Ohira, K. Ogino, and T. Tanigawa, "The effects of aroma foot massage on blood pressure and anxiety in Japanese community-dwelling men and women: a crossover randomized controlled trial," PLOS One, vol. 11, no. 3, Article ID e0151712, 2016.

[48] N. Pasyar, M. Rambod, and F. R. Kahkhaee, "The effect of foot massage on pain intensity and anxiety in patients having undergone a tibial shaft fracture surgery: a randomized clinical trial," Journal of Orthopaedic Trauma, vol. 32, no. 12, pp. e482-e486, 2018.

[49] k. Oshvandi, S. Abdi, A. Karampourian, A. Moghimbaghi, and S. Homayonfar, "The effect of foot massage on quality of sleep in ischemic heart disease patients hospitalized in CCU," Iranian Journal of Critical Care Nursing, vol. 7, no. 2, pp. 66-73, 2014.

[50] T. Bahrami, N. Rejeh, M. Heravi Karimooi, S. D. Tadrisi, and M. Vaismoradi, "The effect of foot reflexology on hospital anxiety and depression in female older adults: a randomized controlled trial," International Journal of Therapeutic Massage \& Bodywork: Research, Education, \& Practice, vol. 12, no. 3, pp. 16-21, 2019.

[51] M. Rambod, N. Pasyar, and M. Shamsadini, "The effect of foot reflexology on fatigue, pain, and sleep quality in lymphoma patients: a clinical trial," European Journal of Oncology Nursing, vol. 43, p. 101678, 2019.

[52] Y. Abbaszadeh, A. Allahbakhshian, A. Seyyedrasooli, P. Sarbakhsh, S. Goljarian, and N. Safaei, "Effects of foot reflexology on anxiety and physiological parameters in patients undergoing coronary artery bypass graft surgery: a clinical trial," Complementary Therapies in Clinical Practice, vol. 31, pp. 220-228, 2018.

[53] S. Saatsaz, R. Rezaei, A. Alipour, and Z. Beheshti, "Massage as adjuvant therapy in the management of post-cesarean pain and anxiety: a randomized clinical trial," Complementary Therapies in Clinical Practice, vol. 24, pp. 92-98, 2016.

[54] G. O. Noh and K. S. Park, "Effects of aroma self-foot reflexology on peripheral neuropathy, peripheral skin temperature, anxiety, and depression in gynaecologic cancer patients undergoing chemotherapy: a randomised controlled trial," European Journal of Oncology Nursing, vol. 42, pp. 82-89, 2019.
[55] M. Soheili, F. Nazari, V. Shaygannejad, and M. Valiani, "A comparison the effects of reflexology and relaxation on the psychological symptoms in women with multiple sclerosis," Journal of Education and Health Promotion, vol. 6, p. 11, 2017.

[56] H. Shahsavari, "The effects of foot reflexology on anxiety and physiological parameters among candidates for bronchoscopy: a randomized controlled trial," European Journal of Integrative Medicine, vol. 12, pp. 177-181, 2017.

[57] F. Ramezanibadr, K. Amini, K. Hossaingholipor, and S. Faghihzadeh, "The impacts of foot reflexology on anxiety among male candidates for coronary angiography: a threegroup single-blind randomized clinical trial," Complementary Therapies in Clinical Practice, vol. 32, pp. 200-204, 2018.

[58] R. Ozturk, U. Sevil, A. Sargin, and M. S. Yucebilgin, "The effects of reflexology on anxiety and pain in patients after abdominal hysterectomy: a randomised controlled trial," Complementary Therapies in Medicine, vol. 36, pp. 107-112, 2018.

[59] M. Bagheri Nesami, S. A. Shorofi, N. Zargar, M. Sohrabi, A. Gholipour Baradari, and A. Khalilian, "The effects of foot reflexology massage on anxiety in patients following coronary artery bypass graft surgery: a randomized controlled trial," Complementary Therapies in Clinical Practice, vol. 20, no. 1, pp. 42-47, 2014.

[60] M. Molavi Vardanjani, N. Masoudi Alavi, N. S. Razavi, M. Aghajani, E. Azizi Fini, and S. M. Vaghefi, "A randomizedcontrolled trial examining the effects of reflexology on anxiety of patients undergoing coronary angiography," Nursing and Midwifery Studies, vol. 2, no. 3, pp. 3-9, 2013.

[61] I. Levy, S. Attias, T. Stern Lavee et al., "The effectiveness of foot reflexology in reducing anxiety and duration of labor in primiparas: an open-label randomized controlled trial," Complementary Therapies in Clinical Practice, vol. 38, Article ID 101085, 2020.

[62] I. Toygar, O. U. Yesilbalkan, Y. G. Malseven, and E. Sonmez, "Effect of reflexology on anxiety and sleep of informal cancer caregiver: randomized controlled trial," Complementary Therapies in Clinical Practice, vol. 39, Article ID 101143, 2020.

[63] C. Y. Li, S. C. Chen, C. Y. Li, M. L. Gau, and C. M. Huang, "Randomised controlled trial of the effectiveness of using foot reflexology to improve quality of sleep amongst Taiwanese postpartum women," Midwifery, vol. 27, no. 2, pp. 181-186, 2011.

[64] A. Samarehfekri, M. Dehghan, M. Arab, and M. R. Ebadzadeh, "Effect of foot reflexology on pain, fatigue, and quality of sleep after kidney transplantation surgery: a parallel randomized controlled trial," Evidence-Based Complementary and Alternative Medicine, vol. 2020, Article ID 5095071, 9 pages, 2020.

[65] H. J. Song, H. Son, H. J. Seo, H. Lee, S. M. Choi, and S. Lee, "Effect of self-administered foot reflexology for symptom management in healthy persons: a systematic review and meta-analysis," Complementary Therapies in Medicine, vol. 23, no. 1, pp. 79-89, 2015.

[66] W. F. Yeung, K. F. Chung, M. M. Poon et al., "Acupressure, reflexology, and auricular acupressure for insomnia: a systematic review of randomized controlled trials," Sleep Medicine, vol. 13, no. 8, pp. 971-984, 2012.

[67] J. Lee, M. Han, Y. Chung, J. Kim, and J. Choi, "Effects of foot reflexology on fatigue, sleep and pain: a systematic review and meta-analysis," Journal of Korean Academy of Nursing, vol. 41, no. 6, pp. 821-833, 2011.

[68] M. Hasanpour, M. M. Mohammadi, and H. Shareinia, "Effects of reflexology on premenstrual syndrome: a systematic review and meta-analysis," BioPsychoSocial Medicine, vol. 13, p. 25, 2019. 
[69] R. Chandrababu, E. L. Rathinasamy, C. Suresh, and J. Ramesh, "Effectiveness of reflexology on anxiety of patients undergoing cardiovascular interventional procedures: a systematic review and meta-analysis of randomized controlled trials," Journal of Advanced Nursing, vol. 75, no. 1, pp. 43-53, 2019.

[70] K. F. Schulz, I. Chalmers, R. J. Hayes, and D. G. Altman, "Empirical evidence of bias: dimensions of methodological quality associated with estimates of treatment effects in controlled trials," JAMA, vol. 273, no. 5, pp. 408-412, 1995.

[71] M. J. Verhoef, A. L. Casebeer, and R. J. Hilsden, “Assessing efficacy of complementary medicine: adding qualitative research methods to the gold standard," The Journal of Alternative and Complementary Medicine, vol. 8, no. 3, pp. 275-281, 2002.

[72] A. Hrobjartsson, F. Emanuelsson, A. S. Skou Thomsen, J. Hilden, and S. Brorson, "Bias due to lack of patient blinding in clinical trials: a systematic review of trials randomizing patients to blind and nonblind sub-studies," International Journal of Epidemiology, vol. 43, no. 4, pp. 1272-1283, 2014.

[73] K. F. Schulz, D. G. Altman, and D. Moher, "Consort 2010 statement: updated guidelines for reporting parallel group randomised trials," Journal of Pharmacology and Pharmacotherapeutics, vol. 1, no. 2, pp. 100-107, 2010. 